- Additional material is published online only. To view please visit the journal online (http://dx.doi.org/10.1136/ injuryprev-2019-043494).

For numbered affiliations see end of article.

Correspondence to Dr Spencer L James, Institute for Health Metrics and Evaluation, University of Washington, Seattle, WA 98121, USA; spencj@uw.edu

Received 29 September 2019 Revised 29 November 2019 Accepted 6 December 2019 Published Online First 24 April 2020
Check for updates

(C) Author(s) (or their employer(s)) 2020. Re-use permitted under CC BY Published by BMJ.

To cite: James SL, Castle CD, Dingels ZV, et al. Inj Prev 2020;26:i96-i114.

\section{(2) OPEN ACCESS \\ Global injury morbidity and mortality from 1990 to 2017: results from the Global Burden of Disease Study 2017}

Spencer L James, ${ }^{1}$ Chris D Castle, ${ }^{1}$ Zachary V Dingels, ${ }^{1}$ Jack T Fox, ${ }^{1}$ Erin B Hamilton, Zichen Liu, ${ }^{1}$ Nicholas L S Roberts, ${ }^{1}$ Dillon O Sylte, ${ }^{1}$ Nathaniel J Henry, ${ }^{1}$ Kate E LeGrand, ${ }^{1}$ Ahmed Abdelalim, ${ }^{2}$ Amir Abdoli, ${ }^{3}$ Ibrahim Abdollahpour, ${ }_{1}^{4}$ Rizwan Suliankatchi Abdulkader, ${ }^{5}$ Aidin Abedi, ${ }^{6}$ Akine Eshete Abosetugn, ${ }^{7}$ Abdelrahman I Abushouk, ${ }^{8}$ Oladimeji M Adebayo, ${ }^{9}$ Marcela Agudelo-Botero, ${ }^{10}$ Tauseef Ahmad, ${ }^{11,12}$ Rushdia Ahmed, ${ }^{13,14}$ Muktar Beshir Ahmed, ${ }^{15}$ Miloud Taki Eddine Aichour, ${ }_{1}^{16}$ Fares Alahdab ${ }_{1}^{17}$ Genet Melak Alamene, ${ }^{18}$ Fahad Mashhour Alanezi, ${ }^{19}$ Animut Alebel, ${ }^{20}$ Niguse Meles Alema, ${ }^{21}$ Suliman A Alghnam, ${ }^{22}$ Samar Al-Hajj, ${ }^{23,24}$ Beriwan Abdulqadir Ali, ${ }^{25,26}$ Saqib Ali, ${ }^{27}$ Mahtab Alikhani, ${ }^{28}$ Cyrus Alinia, ${ }^{29}$ Vahid Alipour, ${ }^{30,31}$ Syed Mohamed Aljunid, ${ }^{32,33}$ Amir Almasi-Hashiani, ${ }^{34}$ Nihad A Almasri, ${ }^{35}$ Khalid Altirkawi, ${ }^{36}$

Yasser Sami Abdeldayem Amer, ${ }_{1}^{37,38}$ Saeed Amini, ${ }^{39}$ Arianna Maever Loreche Amit, ${ }_{1}^{40,41}$ Catalina Liliana Andrei, ${ }^{42}$ Alireza Ansari-Moghaddam ${ }_{1}^{43}$ Carl Abelardo T Antonio, ${ }^{44,45}$ Seth Christopher Yaw Appiah, ${ }^{46,47}$ Jalal Arabloo, ${ }^{30}$ Morteza Arab-Zozani, ${ }^{48}$ Zohreh Arefi, ${ }^{49}$ Olatunde Aremu ${ }^{50}$ Filippo Ariani, ${ }^{51}$ Amit Arora, ${ }^{52,53}$ Malke Asaad, ${ }^{54}$ Babak Asghari, ${ }^{55}$ Nefsu Awoke, ${ }^{56}$ Beatriz Paulina Ayala Quintanilla, ${ }^{57,58}$ Getinet Ayano ${ }^{59}$ Martin Amogre Ayanore, ${ }^{60}$ Samad Azari, ${ }^{30}$ Ghasem Azarian, ${ }^{61}$ Alaa Badawi, ${ }^{62,63}$ Ashish D Badiye, ${ }^{64}$ Eleni Bagli, ${ }^{65,66}$ Atif Amin Baig, ${ }^{67,68}$ Mohan Bairwa, ${ }_{1}^{69,70}$ Ahad Bakhtiari, ${ }^{71}$ Arun Balachandran, ${ }^{72,73}$ Maciej Banach, ${ }^{74,75}$ Srikanta K Banerjee, ${ }^{76}$ Palash Chandra Banik, ${ }^{77}$ Amrit Banstola, ${ }^{78}$ Suzanne Lyn Barker-Collo, ${ }^{79}$ Till Winfried Bärnighausen, ${ }^{80,81}$ Lope H Barrero, ${ }^{82}$ Akbar Barzegar, ${ }^{83}$ Mohsen Bayati, ${ }^{84}$ Bayisa Abdissa Baye, ${ }_{1}^{85}$ Neeraj Bedi, ${ }^{86,87}$ Masoud Behzadifar ${ }^{88}$ Tariku Tesfaye Bekuma, ${ }^{89}$ Habte Belete, ${ }^{90}$ Corina Benjet ${ }_{1}{ }^{91}$ Derrick A Bennett, ${ }^{92}$ Isabela M Bensenor, ${ }^{93}$ Kidanemaryam Berhe, ${ }^{94}$ Pankaj Bhardwaj ${ }_{1}^{95,96}$ Anusha Ganapati Bhat, ${ }^{97}$ Krittika Bhattacharyya, ${ }^{98,99}$ Sadia Bibi, ${ }^{100}$ Ali Bijani, ${ }^{101}$ Muhammad Shahdaat Bin Sayeed, ${ }^{102,103}$ Guilherme Borges, ${ }^{91}$ Antonio Maria Borzì, ${ }^{104}$ Soufiane Boufous, ${ }^{105}$ Alexandra Brazinova, ${ }^{106}$ Nikolay Ivanovich Briko, ${ }^{107}$ Shyam S Budhathoki, ${ }^{108}$ Josip Car, ${ }_{109,110}$ Rosario Cárdenas, ${ }_{111}^{11}$ Félix Carvalho, ${ }_{112}^{112}$ João Mauricio Castaldelli-Maia, ${ }^{113}$ Carlos A Castañeda-Orjuela, ${ }^{114,115}$ Giulio Castelpietra ${ }^{116,117}$ Ferrán Catalá-López, ${ }^{18,119}$ Ester Cerin, ${ }^{120,121}$ Joht S Chandan, ${ }^{122}$ Wagaye Fentahun Chanie, ${ }^{123}$ Soosanna Kumary Chattu, ${ }^{124}$ Vijay Kumar Chattu, ${ }^{125}$ Irini Chatziralli, ${ }^{126,127}$ Neha Chaudhary, ${ }^{128,129}$ Daniel Youngwhan Cho, ${ }^{130}$ Mohiuddin Ahsanul Kabir Chowdhury, ${ }^{131,132}$ Dinh-Toi Chu, ${ }^{133}$ Samantha M Colquhoun, ${ }^{134}$ Maria-Magdalena Constantin, ${ }^{135,136}$ Vera M Costa, ${ }^{112}$ Giovanni Damiani, ${ }^{137,138}$ Ahmad Daryani, ${ }^{139}$ Claudio Alberto Dávila-Cervantes, ${ }_{1}^{140}$ Feleke Mekonnen Demeke, ${ }^{141}$ Asmamaw Bizuneh Demis, ${ }_{1}^{142,143}$ Gebre Teklemariam Demoz, ${ }_{1}^{144,145}$ Desalegn Getnet Demsie, ${ }^{21}$ Afshin Derakhshani, ${ }^{146}$ Kebede Deribe, ${ }_{1}^{147,148}$ Rupak Desai, ${ }^{149}$ Mostafa Dianati Nasab, ${ }^{150}$ Diana Dias da Silva, ${ }^{151}$ Zahra Sadat Dibaji Forooshani, ${ }^{152}$ Kerrie E Doyle, ${ }^{153}$ Tim Robert Driscoll, ${ }^{154}$ 
Eleonora Dubljanin, ${ }^{155}$ Bereket Duko Adema, ${ }^{156,157}$ Arielle Wilder Eagan, ${ }^{158,159}$ Aziz Eftekhari, ${ }^{160,161}$ Elham Ehsani-Chimeh, ${ }^{162}$ Maysaa El Sayed Zaki, ${ }^{163}$ Demelash Abewa Elemineh, ${ }^{164}$ Shaimaa I El-Jaafary, ${ }^{2}$ Ziad El-Khatib, ${ }^{165,166}$ Christian Lycke Ellingsen, ${ }^{167,168}$ Mohammad Hassan Emamian, ${ }^{169}$ Daniel Adane Endalew, ${ }^{170}$ Sharareh Eskandarieh, ${ }^{171}$ Pawan Sirwan Faris, ${ }^{172,173}$ Andre Faro, ${ }^{174}$ Farshad Farzadfar, ${ }^{175}$ Yousef Fatahi, ${ }^{176}$ Wubalem Fekadu, ${ }^{90,177}$ Tomas Y Ferede, ${ }^{178}$ Seyed-Mohammad Fereshtehnejad, ${ }^{179,180}$ Eduarda Fernandes, ${ }^{181}$ Pietro Ferrara, ${ }^{182}$ Garumma Tolu Feyissa, ${ }^{183}$ Irina Filip, ${ }^{184,185}$ Florian Fischer, ${ }^{186}$ Morenike Oluwatoyin Folayan, ${ }^{187}$ Masoud Foroutan, ${ }^{188}$ Joel Msafiri Francis, ${ }^{189}$ Richard Charles Franklin, ${ }^{190,191}$ Takeshi Fukumoto, ${ }^{192,193}$ Biniyam Sahiledengle Geberemariyam, ${ }^{194}$ Abadi Kahsu Gebre, ${ }^{195}$ Ketema Bizuwork Gebremedhin, ${ }^{196}$ Gebreamlak Gebremedhn Gebremeskel, ${ }^{197,198}$ Berhe Gebremichael, ${ }^{199}$ Getnet Azeze Gedefaw, ${ }^{200,201}$ Birhanu Geta, ${ }^{202}$ Mansour Ghafourifard, ${ }^{203}$ Farhad Ghamari, ${ }^{204}$ Ahmad Ghashghaee, ${ }^{205}$ Asadollah Gholamian, ${ }^{206,207}$ Tiffany K Gill, ${ }^{208}$ Alessandra C Goulart, ${ }^{93,209}$ Ayman Grada, ${ }^{210}$ Michal Grivna, ${ }^{211}$ Mohammed Ibrahim Mohialdeen Gubari, ${ }^{212}$ Rafael Alves Guimarães, ${ }^{213}$ Yuming Guo, ${ }^{214,215}$ Gaurav Gupta, ${ }^{216}$ Juanita A Haagsma, ${ }^{217}$ Nima Hafezi-Nejad, ${ }^{218,219}$ Hassan Haghparast Bidgoli, ${ }^{220}$ Brian James Hall, ${ }^{221}$ Randah R Hamadeh, ${ }^{222}$ Samer Hamidi, ${ }^{223}$ Josep Maria Haro, ${ }^{224,225}$ Md Mehedi Hasan, ${ }^{226}$ Amir Hasanzadeh, ${ }^{227,228}$ Soheil Hassanipour, ${ }^{229}$ Hadi Hassankhani, ${ }^{230,231}$ Hamid Yimam Hassen, ${ }^{232,233}$ Rasmus Havmoeller, ${ }^{234}$ Khezar Hayat $_{1}^{235,236}$ Delia Hendrie, ${ }^{59}$ Fatemeh Heydarpour, $^{237}$ Martha Híjar, $^{238,239}$ Hung Chak Ho, ${ }^{240}$ Chi Linh Hoang, ${ }^{241}$ Michael K Hole, ${ }^{242}$ Ramesh Holla, ${ }^{243}$ Naznin Hossain, ${ }^{244,245}$ Mehdi Hosseinzadeh, ${ }^{246,247}$ Sorin Hostiuc, ${ }^{248,249}$ Guoqing Hu, ${ }^{250}$ Segun Emmanuel Ibitoye, ${ }^{251}$ Olayinka Stephen Ilesanmi, ${ }^{252}$ Irena Ilic, ${ }^{155}$ Milena D Ilic, ${ }_{1}^{253}$ Leeberk Raja Inbaraj, ${ }_{1}^{254}$ Endang Indriasih, ${ }^{255}$ Seyed Sina Naghibi Irvani, ${ }^{256}$ Sheikh Mohammed Shariful Islam, ${ }^{257,258}$ M Mofizul Islam, ${ }^{259}$ Rebecca Q Ivers, ${ }^{260}$ Kathryn H Jacobsen, ${ }^{261}$ Mohammad Ali Jahani, ${ }^{262}$ Nader Jahanmehr, ${ }^{263,264}$ Mihajlo Jakovljevic, ${ }^{265}$ Farzad Jalilian, ${ }^{266}$ Sudha Jayaraman, ${ }^{267}$ Achala Upendra Jayatilleke, ${ }^{268,269}$ Ravi Prakash Jha, ${ }^{270}$ Yetunde O John-Akinola, ${ }^{251}$ Jost B Jonas, ${ }^{271,272}$ Nitin Joseph, ${ }^{273}$ Farahnaz Joukar, ${ }_{1}^{229}$ Jacek Jerzy Jozwiak, ${ }^{274}$ Suresh Banayya Jungari, ${ }^{275}$ Mikk Jürisson, ${ }^{276}$ Ali Kabir, ${ }^{277}$ Rajendra Kadel, ${ }^{278}$ Amaha Kahsay, ${ }^{94}$ Leila R Kalankesh, ${ }^{279}$ Rohollah Kalhor, ${ }^{280,281}$ Teshome Abegaz Kamil, ${ }^{282}$ Tanuj Kanchan, ${ }^{283}$ Neeti Kapoor, ${ }^{64}$ Manoochehr Karami, ${ }^{284}$ Amir Kasaeian, ${ }^{285,286}$ Hagazi Gebremedhin Kassaye, ${ }^{21}$ Taras Kavetskyy, ${ }^{287,288}$ Hafte Kahsay Kebede, ${ }^{289}$ Peter Njenga Keiyoro, ${ }^{290}$ Abraham Getachew Kelbore, ${ }^{291}$ Bayew Kelkay, ${ }^{292}$ Yousef Saleh Khader, ${ }^{293}$ Morteza Abdullatif Khafaie, ${ }^{294}$ Nauman Khalid, ${ }^{295}$ Ibrahim A Khalil, ${ }^{296}$ Rovshan Khalilov, ${ }^{297}$ Mohammad Khammarnia, ${ }^{298}$ Ejaz Ahmad Khan, ${ }^{299}$ Maseer Khan, ${ }^{300}$ Tripti Khanna, ${ }^{301,302}$ Habibolah Khazaie, ${ }^{303}$ Fatemeh Khosravi Shadmani, ${ }^{304}$ Roba Khundkar, ${ }^{305}$ Daniel N Kiirithio, ${ }^{306}$ Young-Eun Kim, ${ }^{307}$ Daniel Kim, ${ }^{308}$ Yun Jin Kim, ${ }^{309}$ Adnan Kisa, ${ }^{310}$ Sezer Kisa, ${ }^{311}$ Hamidreza Komaki, ${ }^{312,313}$ Shivakumar K M Kondlahalli, ${ }^{314}$ Vladimir Andreevich Korshunov, ${ }^{107}$ Ai Koyanagi, ${ }^{315,316}$ Moritz U G Kraemer, ${ }^{317,318}$ Kewal Krishan, ${ }^{319}$ Burcu Kucuk Bicer, $^{320,321}$ Nuworza Kugbey, ${ }^{322,323}$ Vivek Kumar, ${ }^{324}$ Nithin Kumar, $^{273}$ G Anil Kumar, ${ }^{325}$ Manasi Kumar, $^{326,327}$ Girikumar Kumaresh, ${ }^{328}$ Om P Kurmi, ${ }^{327,329}$ Oluwatosin Kuti, ${ }^{330}$ Carlo La Vecchia, ${ }^{331}$ Faris Hasan Lami, ${ }^{332}$ Prabhat Lamichhane, ${ }^{333}$ Justin J Lang, ${ }^{334}$ Van C Lansingh, ${ }^{335,336}$ Dennis Odai Laryea, ${ }^{337}$ Savita Lasrado, ${ }^{338}$ Arman Latifi, ${ }^{339}$ Paolo Lauriola, ${ }^{340}$ Janet L Leasher, ${ }^{341}$ Shaun Wen Huey Lee, ${ }^{342,343}$ Tsegaye Lolaso Lenjebo, $^{344}$ Miriam Levi, ${ }^{51,345}$ Shanshan Li, ${ }^{214}$ Shai Linn, ${ }^{346}$ Xuefeng Liu, ${ }^{347}$ Alan D Lopez, ${ }^{1,348,349}$ Paulo A Lotufo, ${ }^{350}$ Raimundas Lunevicius, ${ }^{351,352}$ Ronan A Lyons, ${ }^{353}$ Mohammed Madadin, ${ }^{354}$ Muhammed Magdy Abd El Razek, ${ }^{355}$ Narayan Bahadur Mahotra, ${ }^{356}$ Marek Majdan, ${ }^{357}$ Azeem Majeed, ${ }^{358}$ Jeadran N Malagon-Rojas, ${ }^{359,360}$ Venkatesh Maled, ${ }^{361,362}$ Reza Malekzadeh, 363,364 Deborah Carvalho Malta, ${ }^{365}$ Navid Manafi, ${ }^{366,367}$ Amir Manafi, ${ }^{368}$ Ana-Laura Manda, ${ }^{369}$ Narayana Manjunatha, ${ }^{370}$ Fariborz Mansour-Ghanaei, ${ }^{229}$ Borhan Mansouri, ${ }^{371}$ Mohammad Ali Mansournia, ${ }^{372}$ Joemer C Maravilla, ${ }^{373}$ Lyn M March, ${ }^{374}$ Amanda J Mason-Jones, ${ }^{375}$ Seyedeh Zahra Masoumi, ${ }^{376}$ Benjamin Ballard Massenburg, ${ }^{130}$ Pallab K Maulik, ${ }^{377,378}$ Gebrekiros Gebremichael Meles, ${ }^{379}$

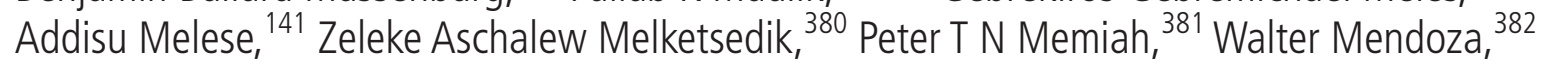


Ritesh G Menezes, ${ }_{1}^{383}$ Meresa Berwo Mengesha, ${ }^{384}$ Melkamu Merid Mengesha, ${ }_{1}^{385}$ Tuomo J Meretoja, ${ }_{1}^{386,387}$ Atte Meretoja, ${ }^{388,389}$ Hayimro Edemealem Merie, ${ }^{164}$ Tomislav Mestrovic, ${ }^{390,391}$ Bartosz Miazgowski, ${ }^{392}$ Tomasz Miazgowski, ${ }^{393}$ Ted R Miller, ${ }^{59,394}$ GK Mini, ${ }^{395,396}$ Andreea Mirica, ${ }_{1}^{397,398}$ Erkin M Mirrakhimov, ${ }_{1}^{399,400}$ Mehdi Mirzaei-Alavijeh, ${ }^{266}$ Prasanna Mithra, ${ }^{273}$ Babak Moazen, ${ }^{401,402}$ Masoud Moghadaszadeh, ${ }^{403,404}$ Efat Mohamadi, ${ }^{405}$ Yousef Mohammad, ${ }^{406}$ Karzan Abdulmuhsin Mohammad, ${ }^{407,408}$ Aso Mohammad Darwesh, ${ }^{409}$ Naser Mohammad Gholi Mezerji, ${ }^{410}$ Abdollah Mohammadian-Hafshejani, ${ }^{411}$ Milad Mohammadoo-Khorasani, ${ }^{412}$ Reza Mohammadpourhodki, ${ }^{413}$ Shafiu Mohammed, ${ }^{80,414}$ Jemal Abdu Mohammed, ${ }^{415}$ Farnam Mohebi, ${ }^{175,416}$ Mariam Molokhia, ${ }^{417}{ }^{2}$ Lorenzo Monasta, ${ }^{418}$ Yoshan Moodley, ${ }^{419}$ Mahmood Moosazadeh, ${ }^{420}$ Masoud Moradi, ${ }^{421}$ Ghobad Moradi, ${ }^{422,423}$ Maziar Moradi-Lakeh, ${ }^{424}$ Farhad Moradpour, ${ }^{422}$ Lidia Morawska, ${ }^{425}$ Ilais Moreno Velásquez, ${ }^{426}$ Naho Morisaki, ${ }^{427}$ Shane Douglas Morrison, ${ }^{130}$ Tilahun Belete Mossie, ${ }^{90}$ Atalay Goshu Muluneh, ${ }^{428}$ Srinivas Murthy, ${ }^{429}$ Kamarul Imran Musa, ${ }^{430}$ Ghulam Mustafa, ${ }^{431,432}$ Ashraf F Nabhan, ${ }^{433,434}$ Ahamarshan Jayaraman Nagarajan, ${ }^{435,436}$ Gurudatta Naik, ${ }^{437}$ Mukhammad David Naimzada, 438,439 Farid Najafi, ${ }^{440}$ Vinay Nangia ${ }^{441}{ }^{4}$ Bruno Ramos Nascimento, ${ }^{442}$ Morteza Naserbakht, ${ }^{424,443}{ }^{\text {Vinod Nayak, }}{ }^{444}$ Duduzile Edith Ndwandwe, ${ }^{445}$ Ionut Negoi, ${ }^{446,447}$ Josephine W Ngunjiri, ${ }^{448}$ Cuong Tat Nguyen, ${ }^{449}$ Huong Lan Thi Nguyen, ${ }^{449}$ Rajan Nikbakhsh, ${ }^{450,451}$ Dina Nur Anggraini Ningrum, ${ }^{452,453}$ Chukwudi A Nnaji, ${ }^{445,454}$ Peter S Nyasulu, ${ }^{455}$ Felix Akpojene Ogbo, ${ }^{112}$ Onome Bright Oghenetega ${ }^{456}$ In-Hwan Oh, ${ }^{457}$ Emmanuel Wandera Okunga, ${ }^{458}$ Andrew T Olagunju, ${ }^{459,460}$ Tinuke O Olagunju, ${ }^{461}$ Ahmed Omar Bali, ${ }^{462}$ Obinna E Onwujekwe, ${ }^{463}$ Kwaku Oppong Asante, ${ }^{464,465}$ Heather M Orpana, ${ }^{466,467}$ Erika Ota, ${ }^{468}$ Nikita Otstavnov, ${ }^{438,469}$ Stanislav S Otstavnov, ${ }^{438,470}$ Mahesh P A, ${ }^{471}$ Jagadish Rao Padubidri, ${ }^{472}$ Smita Pakhale, ${ }^{473}$ Keyvan Pakshir, ${ }^{474}$ Songhomitra Panda-Jonas, ${ }^{475}$ Eun-Kee Park, ${ }^{476}$

Sangram Kishor Patel, ${ }^{477,478}$ Ashish Pathak, ${ }^{165,479}$ Sanghamitra Pati, ${ }^{480}$ George C Patton, ${ }^{481,482}$ Kebreab Paulos, ${ }^{483}$ Amy E Peden, ${ }^{191,484}$ Veincent Christian Filipino Pepito, ${ }^{485}$ Jeevan Pereira, ${ }^{486}$ Hai Quang Pham, ${ }^{449}$ Michael R Phillips, ${ }^{487,488}$ Marina Pinheiro, ${ }^{489}$ Roman V Polibin, 490 Suzanne Polinder, ${ }^{217}$ Hossein Poustchi, ${ }^{363}$ Swayam Prakash, ${ }^{491}$ Dimas Ria Angga Pribadi, ${ }^{492}$ Parul Puri, ${ }^{493}$ Zahiruddin Quazi Syed, ${ }^{96}$ Mohammad Rabiee, ${ }^{494}$ Navid Rabiee, ${ }^{495}$ Amir Radfar, ${ }^{496,497}$ Anwar Rafay, ${ }^{498}$ Ata Rafiee, ${ }^{499}$ Alireza Rafiei, ${ }^{500,501}$ Fakher Rahim, ${ }^{502,503}$ Siavash Rahimi, ${ }^{504}$ Vafa Rahimi-Movaghar, ${ }^{505}$ Muhammad Aziz Rahman, ${ }^{506,507}$ Ali Rajabpour-Sanati, ${ }^{508}$ Fatemeh Rajati, ${ }^{421}$ Ivo Rakovac, ${ }^{509}$ Kavitha Ranganathan, ${ }^{510}$ Sowmya J Rao, ${ }^{511}$ Vahid Rashedi, ${ }^{512}$ Prateek Rastogi, ${ }^{513}$ Priya Rathi, ${ }^{514}$ Salman Rawaf, ${ }^{358,515}$ Lal Rawal, ${ }^{516}$ Reza Rawassizadeh, ${ }^{517}$ Vishnu Renjith, ${ }^{518}$ Andre M N Renzaho, ${ }^{519,520}$ Serge Resnikoff, ${ }^{521}$ Aziz Rezapour, ${ }^{522}$ Ana Isabel Ribeiro, ${ }^{523}$ Jennifer Rickard, ${ }^{524}$ Carlos Miguel Rios González, ${ }^{526,527}$ Luca Ronfani, ${ }^{418}$ Gholamreza Roshandel, ${ }^{363,528}$ Anas M Saad, ${ }^{529}$ Yogesh Damodar Sabde, ${ }^{530}$ Siamak Sabour, ${ }^{531}$ Basema Saddik, ${ }^{532}$ Saeed Safari, ${ }^{533}$ Roya Safari-Faramani, ${ }^{534}$ Hamid Safarpour, ${ }^{535}$ Mahdi Safdarian, ${ }^{505,536}$ S Mohammad Sajadi, ${ }^{537}$ Payman Salamati, ${ }^{505}$ Farkhonde Salehi, ${ }^{538}$ Saleh Salehi Zahabi, ${ }^{539,540}$ Marwa R Rashad Salem, ${ }^{541}$ Hosni Salem, ${ }^{542}$ Omar Salman, ${ }^{543,544}$ Inbal Salz, ${ }^{545}$ Abdallah M Samy, ${ }^{546}$ Juan Sanabria, ${ }^{547,548}$ Lidia Sanchez Riera, ${ }^{549,550}$ Milena M Santric Milicevic, ${ }^{551,552}$ Abdur Razzaque Sarker, ${ }^{553}$ Arash Sarveazad, ${ }^{554}$ Brijesh Sathian, ${ }^{555,556}$ Monika Sawhney ${ }^{557}$ Susan M Sawyer, ${ }^{558,559}$ Sonia Saxena, ${ }^{560}$ Mehdi Sayyah, ${ }^{561}$ David C Schwebel, ${ }^{562}$ Soraya Seedat, ${ }^{563}$ Subramanian Senthilkumaran, ${ }^{564}$ Sadaf G Sepanlou, ${ }^{363,364}$ Seyedmojtaba Seyedmousavi, ${ }^{565}$ Feng Sha, ${ }^{566}$ Faramarz Shaahmadi, ${ }^{567}$ Saeed Shahabi, ${ }^{568}$ Masood Ali Shaikh, ${ }^{569}$ Mehran Shams-Beyranvand, ${ }^{570}$ Morteza Shamsizadeh, ${ }^{571}$ Mahdi Sharif-Alhoseini, ${ }^{505}$ Hamid Sharifi, ${ }^{572}$ Aziz Sheikh, ${ }^{573,574}$ Mika Shigematsu, ${ }^{575}$ Jae II Shin ${ }^{576,577}$ Rahman Shiri, ${ }^{578}$ Soraya Siabani, ${ }^{579,580}$ Inga Dora Sigfusdottir, ${ }^{581,582}$ Pankaj Kumar Singh, ${ }^{583}$ Jasvinder A Singh, ${ }^{584,585}$ Dhirendra Narain Sinha, ${ }^{586,587}$ Catalin-Gabriel Smarandache, ${ }^{588,589}$ Emma U R Smith, ${ }^{590,591}$ Amin Soheili, ${ }^{592,593}$ Bija Soleymani, $^{237}$ Ali Reza Soltanian, ${ }^{594}$ Joan B Soriano, ${ }^{595,596}$ Muluken Bekele Sorrie, ${ }^{597}$ Ireneous N Soyiri, ${ }^{598,599}$ Dan J Stein, ${ }^{600,601}$ Mark A Stokes, ${ }^{602}$ Mu'awiyyah Babale Sufiyan, ${ }^{603}$ Hafiz Ansar Rasul Suleria, ${ }^{604}$ Bryan L Sykes, ${ }^{605}$ Rafael Tabarés-Seisdedos, ${ }^{606,607}$ Karen M Tabb, ${ }^{608}$ Biruk Wogayehu Taddele, ${ }^{609}$ 
Degena Bahrey Tadesse, ${ }^{197,610}$ Animut Tagele Tamiru, ${ }^{611}$ Ingan Ukur Tarigan, ${ }^{255}$ Yonatal Mesfin Tefera, ${ }^{612,613}$ Arash Tehrani-Banihashemi, ${ }^{424,614}$ Merhawi Gebremedhin Tekle, ${ }^{199}$ Gebretsadkan Hintsa Tekulu, ${ }^{615}$

Ayenew Kassie Tesema ${ }_{1}^{616}$ Berhe Etsay Tesfay ${ }_{1}^{617}$ Rekha Thapar, ${ }^{273}$ Asres Bedaso Tilahune, ${ }^{618}$ Kenean Getaneh Tlaye, ${ }^{142}$ Hamid Reza Tohidinik, ${ }^{372,572}$ Roman Topor-Madry, ${ }_{1}^{619,620}$ Bach Xuan Tran, ${ }^{621}$ Khanh Bao Tran ${ }^{622,623}$ Jaya Prasad Tripathy, ${ }_{1}^{624}$ Alexander C Tsai, ${ }_{1}^{625,626}$ Lorainne Tudor Car, ${ }_{1}^{627}$ Saif Ullah, ${ }^{628}$ Irfan Ullah, ${ }^{629,630}$ Maida Umar, ${ }^{631}$ Bhaskaran Unnikrishnan, ${ }^{273}$ Era Upadhyay, ${ }^{632}$ Olalekan A Uthman, ${ }_{1}^{633}$ Pascual R Valdez, ${ }^{634,635}$ Tommi Juhani Vasankari, ${ }^{636}$ Narayanaswamy Venketasubramanian, ${ }^{637,638}$ Francesco S Violante ${ }_{1}^{639,640}$ Vasily Vlassov ${ }^{641}$ Yasir Waheed ${ }^{642}$ Girmay Teklay Weldesamuel, ${ }^{197}$ Andrea Werdecker ${ }_{1}^{643,644}$ Taweewat Wiangkham, ${ }^{645}$ Haileab Fekadu Wolde, ${ }_{1}^{428}$ Dawit Habte Woldeyes, ${ }_{1}^{646}$ Dawit Zewdu Wondafrash, ${ }^{647,648}$ Temesgen Gebeyehu Wondmeneh, ${ }^{415}$ Adam Belay Wondmieneh, ${ }^{196,649}$ Ai-Min Wu, ${ }_{1}^{650}$ Rajaram Yadav, ${ }^{493}$ Ali Yadollahpour, ${ }^{651}$ Yuichiro Yano, ${ }^{652}$ Sanni Yaya ${ }^{653}$ Vahid Yazdi-Feyzabadi, ${ }^{654,655}$ Paul Yip ${ }_{1}^{656,657}$ Engida Yisma, ${ }^{658}$ Naohiro Yonemoto, ${ }^{659}$ Seok-Jun Yoon, ${ }^{307}$ Yoosik Youm, ${ }^{660}$ Mustafa Z Younis, ${ }^{661,662}$ Zabihollah Yousefi, ${ }^{663,664}$ Yong Yu, ${ }^{665}$ Chuanhua Yu ${ }_{1}^{666,667}$ Hasan Yusefzadeh, ${ }^{29}$ Telma Zahirian Moghadam, ${ }^{30,668}$ Zoubida Zaidi, ${ }^{669}$ Sojib Bin Zaman, ${ }^{131,670}$ Mohammad Zamani, ${ }^{671}$ Maryam Zamanian, ${ }^{34}$ Hamed Zandian, ${ }^{668,672}$ Ahmad Zarei, ${ }^{673}$ Fatemeh Zare, ${ }^{674}$ Zhi-Jiang Zhang, ${ }^{675}$ Y Yunquan Zhang, ${ }^{676,677}$ Sanjay Zodpey ${ }_{1}^{678}$ Lalit Dandona, ${ }^{1,325,349}$ Rakhi Dandona, ${ }^{1,325}$ Louisa Degenhardt, ${ }^{1,679}$ Samath Dhamminda Dharmaratne, ${ }^{1,349,680}$ Simon I Hay, ${ }^{1,349}$ Ali H Mokdad, ${ }_{1}^{1,349}$ Robert C Reiner Jr, ${ }^{1,349}$ Benn Sartorius, ${ }^{349,681}$ Theo Vos ${ }^{1,349}$

\section{SUMMARY}

Background Past research in population health trends has shown that injuries form a substantial burden of population health loss. Regular updates to injury burden assessments are critical. We report Global Burden of Disease (GBD) 2017 Study estimates on morbidity and mortality for all injuries.

Methods We reviewed results for injuries from the GBD 2017 study. GBD 2017 measured injury-specific mortality and years of life lost (YLLs) using the Cause of Death Ensemble model. To measure non-fatal injuries, GBD 2017 modelled injury-specific incidence and converted this to prevalence and years lived with disability (YLDS). YLLs and YLDs were summed to calculate disability-adjusted life years (DALYs).

Findings In 1990, there were 4260493 (4 085700 to 4396 138) injury deaths, which increased to 4484722 (4 332010 to 4585 554) deaths in 2017, while age-standardised mortality decreased from 1079 (1073 to 1086) to 738 (730 to 745 ) per 100000 . In 1990, there were 354064302 (95\% uncertainty interval: 338174876 to 371610802 ) new cases of injury globally, which increased to 520710288 (493 430247 to 547988 635) new cases in 2017. During this time, agestandardised incidence decreased non-significantly from 6824 (6534 to 7147) to 6763 (6412 to 7118) per 100000 . Between 1990 and 2017, age-standardised DALYs decreased from 4947 (4655 to 5233) per 100000 to 3267 (3058 to 3505).

Interpretation Injuries are an important cause of health loss globally, though mortality has declined between 1990 and 2017. Future research in injury burden should focus on prevention in highburden populations, improving data collection and ensuring access to medical care.

\section{INTRODUCTION}

Injury burden assessments are a critical component of population health measurement. Across the global landscape of population health research, injuries are unique in that they are almost universally avertable yet can cause death or disability at any age. Even common injuries such as concussion resulting from falls, violence or road injuries may cause longer term sequelae, and injuries such as spinal cord injuries or limb amputations can cause long-term disability. ${ }^{1}$ As a result, injuries are recognised as being a source of lost health and human capital that could be averted with improved safety and prevention programmes as well as ensuring access to care resources. ${ }^{2}$ Across geographies, certain injuries such as envenomation may be relevant in specific locations where venomous creatures live, while injuries such as those occurring from adverse medical events are an increasing area of research in higher income areas of the world. ${ }^{3-5}$ Bolstering such programmes, however, requires detailed measurement of when, where and to whom injuries are occurring, necessitating focused research studies to add insight and context to broader geographical trends. Across all domains of injury prevention research, it is important to measure the causes of injury, such as road injuries, and the resulting disability, such as fractures, burns or traumatic brain injury, that can occur as a result. Such detailed measurement lends perspective for understanding burden and anticipating resources needed to care for and hopefully prevent future injury burden. Detailed measurements and assessments of this nature are critical for empowering policy makers and health system planners to appropriately plan and invest for mitigating future health loss from injuries. Reducing injury burden is an important component in global efforts such as the Sustainable Development Goal 3 to 'ensure healthy lives and promote wellbeing for all at all ages'. ${ }^{6}$

While some research has focused on a certain type of injury or outcome from injury or specific area of the world, ${ }^{7-10}$ it has become important in an era of more sophisticated population health measurement to measure health loss from injuries comprehensively with detailed fatal and non-fatal estimates for different ages, sexes, across time periods and accounting for multiple different types of morbidity that can occur in an injury. Previously published literature on global injury burden through 2015 has provided comprehensive measurements of health loss due to injuries but still require regular updates to help inform research and policy, as new years of estimates are 
added and as new injuries and injury outcomes are incorporated. ${ }^{11}$ Comprehensive research of this nature shows how injury burden varies dynamically by age, sex, year, area of the world and type of injury, and hence, it is important to maintain close monitoring of injury burden every year in all parts of the world. In addition, as new datasets and statistical modelling methods become available, producing regular updates to burden estimation also ensures that results are as accurate as possible.

While the burden of injuries is widely studied and monitored through various methods of research, the Global Burden of Diseases, Injuries, and Risk Factors (GBD) Study is the only study framework that routinely provides estimates of morbidity and mortality from an exhaustive list of injuries in all areas of the world across ages and sexes. The most recent update to GBD was published in 2018 and provided morbidity and mortality estimates for 30 mutually exclusive causes of injury for 195 countries from 1990 to $2017 .^{12-17}$ As part of this regular update, new datasets on cause of death and incidence are incorporated into the study, and additional geographical detail is added to better measure heterogeneity in burden estimates at a subnational level. In addition, updates such as reporting both nature of injury and cause of injury (described in more detail below) are incorporated. In this study, we describe key components in the GBD injury methodology and provide results from key trends in injury burden in terms of incidence, prevalence, years lived with disability (YLDs), cause-specific mortality, years of life lost (YLLs) and disability-adjusted life years (DALYs) by country, age groups, sex, year and injury type.

\section{METHODS}

The methods and results in this study are the same as are provided in GBD capstone publications, and a detailed description of GBD data and methods used for all processes related to GBD 2017 is provided in associated studies. ${ }^{12-17}$ Overall, GBD methods are also summarised in online supplementary appendix 1. Below, we summarise the specific methods used for measurement of injuries morbidity and mortality in GBD 2017.

\section{Key components of GBD study design}

The GBD study incorporates several key components to allow for internally consistent estimates across all burden measures and metrics. First, population is measured to ensure consistent denominators for all population-level measurement. Second, allcause mortality is measured using demographic methods. Third, cause-specific mortality for a mutually exclusive, collectively exhaustive hierarchy of diseases and injuries is measured, such that every death has one underlying cause of death and such that estimates for every possible cause of death are included, which requires the use of residual causes like 'other transport injuries'. This results in the sum of cause-specific mortality equalling total all-cause mortality. Fourth, non-fatal health loss is measured for individuals living with a disease or injury that detracts from their full health status. Fifth, a composite measure of mortality and morbidity is computed. These steps are conducted within an age, sex and location hierarchy constructed such that demographic detail is available but where all estimates are internally consistent with all other estimates. GBD produces estimates for all causes, ages, sexes, years and locations. Risk factors and attributable burden for different are also measured, but those results are not included in this study.

\section{Case definition and cause hierarchy}

The GBD case definition for an injury death is a death where the injury was the underlying cause of death. For example, if an individual falls on ice and sustains an epidural haematoma and dies after a seizure, the fall is the underlying cause. If an individual sustains a myocardial infarction and then falls and sustains the same epidural haematoma, then the myocardial infarction is the underlying cause of death. For non-fatal injuries, we define a case as an injury that warranted medical care. For example, if an individual slips and falls but does not sustain any bodily injury, it is not considered an injury. Online supplementary appendix table 1 provides the International Classification of Disease (ICD) codes used to identify causes of injury.

\section{Cause-specific mortality estimation}

Cause-specific mortality from injuries is measured using the Cause of Death Ensemble model (CODEm). CODEm is described in more detail elsewhere; a summary of its use for injuries is as follows. ${ }^{18}$ First, all available data that can be used for cause of death estimation are identified. For injuries, this includes vital registration, verbal autopsy, police records, mortuary data and census data. These data are processed for use in the GBD cause and demographic hierarchy via a series of data processing steps including a process whereby ill-defined causes of death are reassigned to true underlying causes of death, which is described in more detail elsewhere but essentially is the process by which illdefined causes of death are reclassified to causes of death in the GBD cause hierarchy. ${ }^{19}{ }^{20} \mathrm{Next}$, a cause-specific mortality model is developed for each one of the 30 different causes of injury. For example, falls are modelled differently than road injuries, though both use the same CODEm modelling architecture. For each cause of injury, covariates that may be associated with the cause are identified and added as candidate covariates. CODEm runs different combinations of models using different covariates and outcome variables, specifically cause fraction models and causespecific mortality rate models. Ensembles of models are also conducted to test performance of overall models formed from submodels. Once all models have been run, the top-performing models are selected based on out-of-sample predictive validity, wherein the model makes predictions on data that were not included in developing the model. The top-performing models are then weighted according to performance, and the final estimates form the penultimate estimate for cause-specific mortality from that injury. Those estimates are then adjusted to fit within the all-cause mortality estimate, so that cause-specific deaths sum up to the overall mortality estimate for each population and demographic. YLLs are computed as the cause-specific mortality rate at a given age multiplied by the residual life expectancy at that age, which is based on the observed maximum global life expectancy.

\section{Non-fatal injury estimation}

Non-fatal injury estimation is also described in more detail in GBD literature. Key components in this process are as follows. First, data on incidence of non-fatal injury causes (eg, road injuries) is obtained from the GBD collaborator network and other injury research groups and researchers around the world. Data are cleaned and organised according to GBD study guidelines. Next, incidence of each cause of injury is modelled in DisMod-MR 2.1, which is a Bayesian meta-analysis tool used extensively in GBD research. Incidence estimates of injuries requiring medical care for each cause of injury then stream through an analytical pipeline. During this process, injury incidence is split into 
inpatient and outpatient to account for the different severity that is expected to occur. The coefficient that determines this split is derived from locations where both inpatient and outpatient data are available. After this, we measure the proportion of each cause of injury that leads to one of 47 different natures of injury using clinical data where both cause and nature are coded as well a Dirichlet statistical modelling process. Based on these steps, the incidence of each cause is also split into incidence of each cause-nature, which is the proportion of a given cause's incidence leading to some specific nature of injury being the most severe injury sustained as estimated by the Dirichlet regression. These estimates are then converted to short-term and long-term injuries based on probability of each injury becoming long term, as determined by long-term follow-up injury surveys. ${ }^{21-27}$ For short-term injuries, incidence is converted to prevalence based on multiplying incidence by an expected duration of injury as determined by physicians and injury experts involved in the GBD study. For long-term injuries, incidence is converted to prevalence using differential equations that take into account the increased mortality for certain types of injury, for example, traumatic brain injury. ${ }^{1}$ Disability weights as derived elsewhere in the GBD study are then used to measure disability based on nature of injury. ${ }^{28}$ These measures are then summed across natures of injury for each cause to calculate YLDs. Each of these steps is conducted for every cause, age, sex, year and location in the GBD study design. Associated literature provides more detail on each of these steps. ${ }^{12-17}$

\section{DALY measurement}

DALYs are calculated by summing YLLs and YLDs for each cause, age, sex, year and location.

\section{Uncertainty measurement}

Uncertainty is measured at each step of the analytical process based on the sample size, SE or original uncertainty interval (UI) from each input to the study. Uncertainty is propagated through each step of the analysis by maintaining distributions of 1000 draws on which each analytical step is conducted. Final 95\% UIs are determined based on the 25th and 975th values of the ordered values across draws.

\section{Code and results}

Steps of the analytical process were conducted in Python version 2.7, Stata V.13.1 or R version 3.3. All steps of the analytical process are available online at ghdx.healthdata.org. This study reports a subset of measures and metrics for every cause of injury. All results and results with additional detail by age, sex, year and location can be downloaded at ghdx.healthdata.org.

\section{Guidelines for Accurate and Transparent Health Estimates Reporting (GATHER) statement}

This study is adherent with guidelines from the GATHER (described in more detail in online supplementary appendix 2). ${ }^{29}$

\section{RESULTS}

Online supplementary appendix table 2 shows age-standardised incidence, prevalence, YLDs, deaths, YLLs and DALYs in 2017 by country as well as percentage change and UI from 1990 for each metric. Online supplementary appendix table 3 shows allage numbers (ie, not divided by population) of incidence, prevalence, YLDs, deaths, YLLs and DALYs in 2017 by country as well as percentage change from 1990 and UI for each metric. In some instances, the UI for the per cent change crosses zero, meaning that statistically there was no significant difference. Online supplementary appendix figures 1-6, show the incidence and mortality from transport injuries, unintentional injuries, and interpersonal violence and self-harm by country for 2017 as well as the percentage change for both incidence and mortality between 1990 and 2017. All other results including age-specific and sex-specific results can be viewed and downloaded via freely and publicly available tools at ghdx.healthdata.org.

\section{Global trends in overall injury burden}

In terms of fatal outcomes, deaths due to all injuries increased from 4260493 (4 085700 to 4396138 ) in 1990 to 4484722 (4 332 010 to 4585 554) in 2017, while YLLs decreased from 232104 206 (219 920058 to 241973733 ) to 195231148 (188 807653 to 199825464$)$ and age-standardised mortality rates decreased from 1079 (1073 to 1086$)$ to 738 (730 to 745) per 100000. In terms of non-fatal outcomes, all-injury incidence (new cases) increased from 354064302 (338 174876 to 371610802 ) in 1990 to 520710288 (493430247 to 547988635 ) in 2017, and YLDs increased from 37452031 (27805854 to 49010103 ) to 57174469 (42073 855 to 75427 036), while age-standardised incidence rates decreased non-significantly from 6824 (6534 to 7147 ) to 6763 (6412 to 7118) per 100 000. In terms of DALYs, age-standardised DALY rates decreased from 4947 (4655 to 5233) per 100000 in 1990 to 3267 (3058 to 3505) in 2017.

Figure 1 shows age-standardised DALY rates by country for 2017. While certain countries-specifically, Syria, Central African Republic and Iraq-have much higher DALY rates than most other countries, there still exists considerable heterogeneity across countries that are not among these countries with the highest burden. South Sudan, Somalia and Yemen have much higher injury burden than much of the rest of the world, for example, with agestandardised DALY rates of 7391.51 per 100000 (6536.44 to 8440.14), 7364.66 per 100000 (6143.11 to 8960.58) and 7297.88 per 100000 (6525.7 to 8438.15), respectively. Papua New Guinea also demonstrates high all-injury burden with 6803.33 DALYs per 100000 (5652.2 to 8040.89) in 2017.

Figure 2 presents deaths as a stacked graph for overall injury groups and population from 1990 to 2017 with labelled fatal discontinuities, defined as changes in deaths due to sudden, unexpected spikes in mortality that depart from the underlying mortality trend. ${ }^{13}$ Although population has steadily increased in the 28 years of the study, deaths per year due to injuries have remained relatively consistent over time. Natural disasters, such as earthquakes, have caused pronounced spikes in unintentional injuries deaths, while conflict and genocide have caused spikes in deaths in the interpersonal violence injury category.

\section{All-injury YLDs and YLLs by country in 2017}

Figure 3 shows the percentage of total all-age, combined-sex YLDs by country in 2017. This figure shows several geographical patterns that help depict the non-fatal burden of injuries globally in terms of their relative contribution to overall disability. First, the percentage of total disability caused by injuries varies widely by country. Mauritius experiences only 3.04\% (2.79\% to $3.29 \%$ ) of non-fatal burden from injuries, while Slovenia experiences $19.11 \%$ (17.11\% to $21.27 \%)$ of non-fatal burden from injuries. In other words, if all disability in these two populations is combined in 2017, there is over sixfold variation in how much of this disability was caused by injuries. These patterns also reflect burden from non-injury conditions, since locations with higher burden from communicable disease may have correspondingly lower proportion due to injuries. As an extension of 


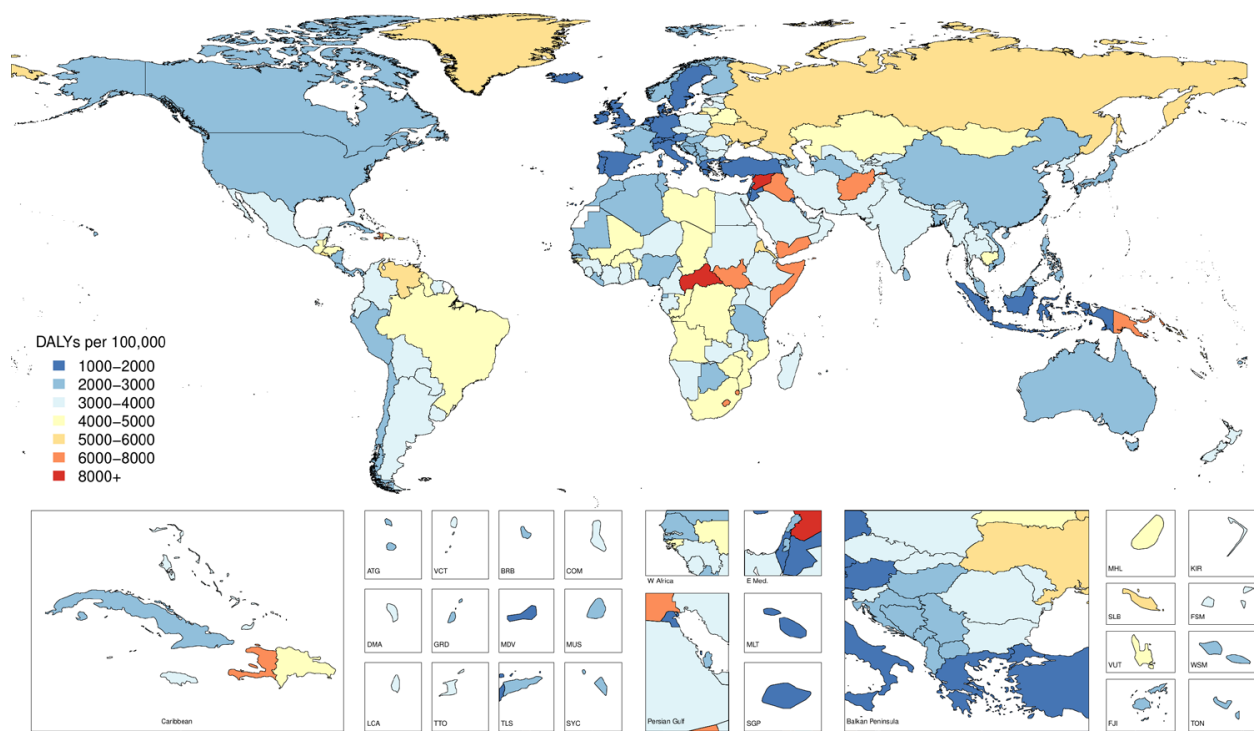

Figure 1 Age-standardised DALY rates by country, 2017. DALYs, disability-adjusted life years.

these geographical trends, this map makes it evident that there are striking regional patterns in non-fatal injury burden. Eastern and Central Europe and Central Asia as well as Australasia have a notably higher percentage of total non-fatal burden from injuries than countries in other regions, while these percentages are relatively lower in most areas of Africa, the Americas and areas of South, East and Southeast Asia. To some extent, this map also reflects the underlying burden from non-injury causes, too, since areas of the world with high non-fatal disability from conditions such as anaemia, communicable diseases and other types of health loss could have correspondingly higher percentages of disability from these conditions instead of injuries. This map also shows examples of positive deviations from global trends; Indonesia, for example, has a relatively low percentage of non-fatal health loss due to injuries compared with many other countries.

Figure 4 similarly shows the percentage of total all-age, combined-sex YLLs by country in 2017. This figure interestingly shows how mortality patterns demonstrate different geographical trends than the non-fatal burden, as depicted in figure 2, though it should be noted that YLLs will also be disproportionately higher in younger populations, all else being equal. In particular, the locations with the highest percentage of YLLs due to injuries are in certain countries in North Africa and the Middle East, including Syria, where 59.51\% (56.59\% to $62.35 \%$ ) of YLLs were due to injuries in 2017, and Iraq, where $41.34 \%$ of YLLs were due to injuries in 2017. Areas of

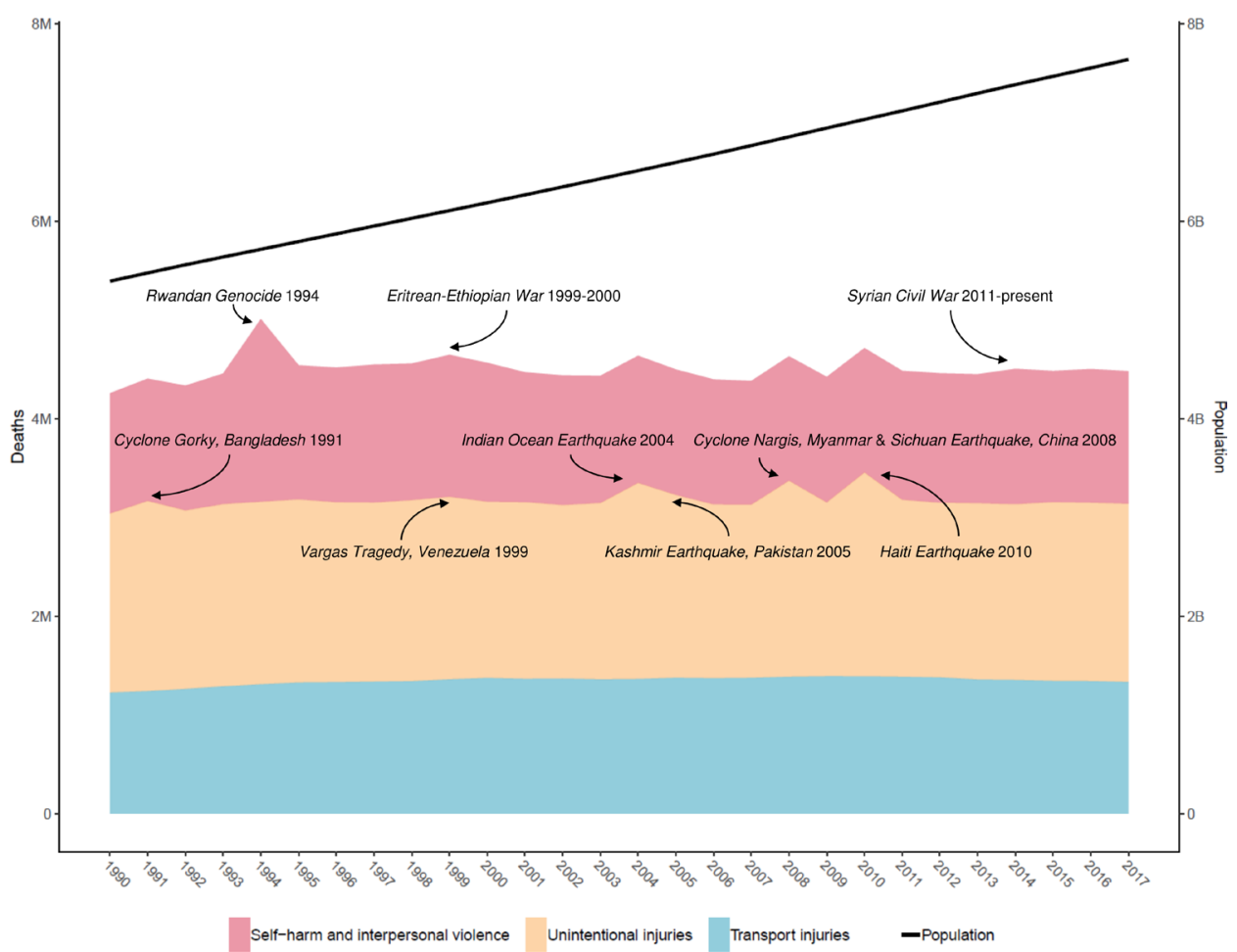

Figure 2 Global deaths for level 2 injuries and population from 1990 to 2017 with labelled fatal discontinuities. 


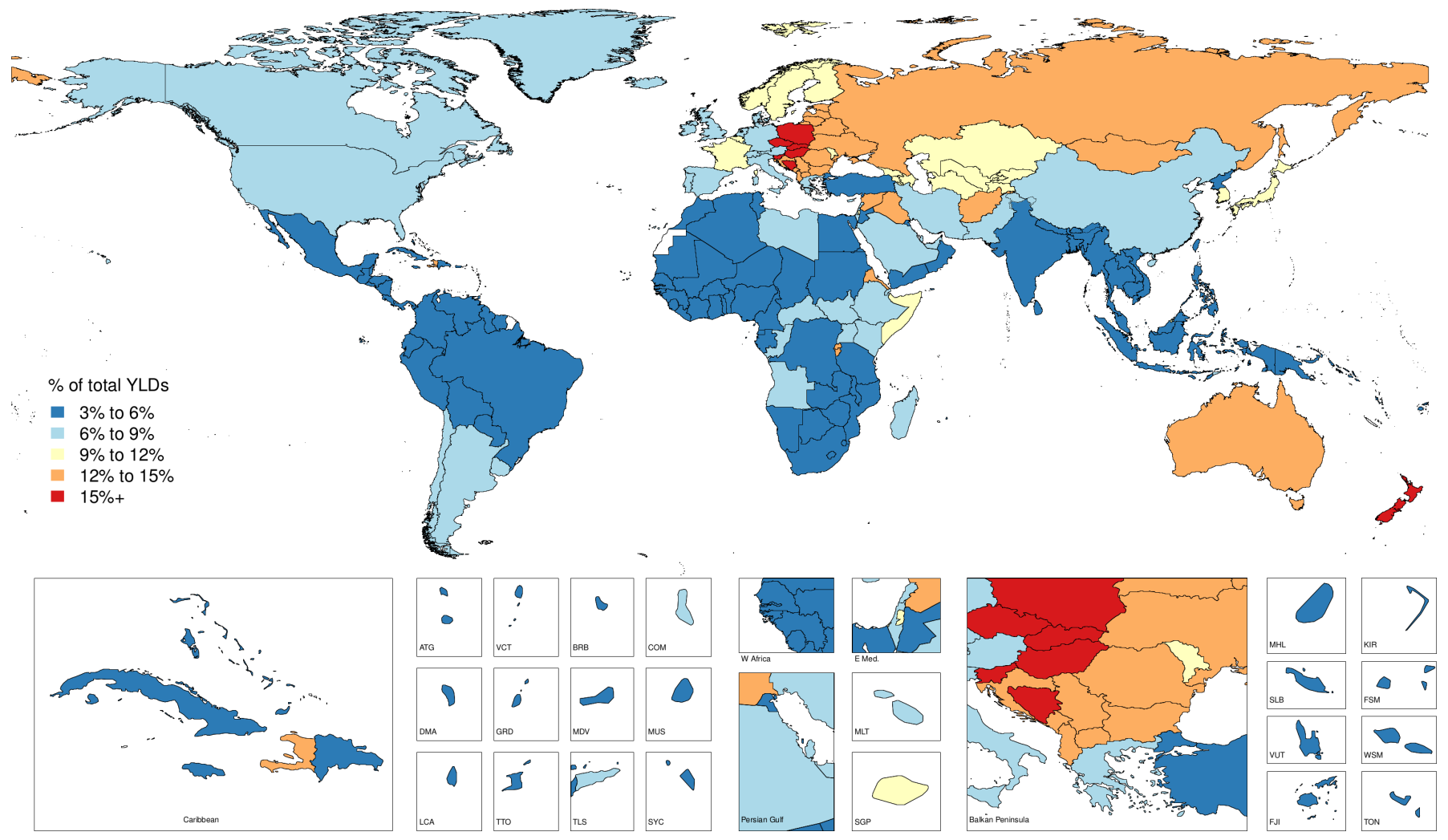

Figure 3 Percentage of YLDs in all ages due to injuries in 2017. YLDs, years lived with disability.

Latin America including Venezuela, Honduras and Belize also have a relatively high percentage of total YLLs due to injuries. Conversely, certain areas of the world also demonstrate a relatively low percentage of total YLLs due to injuries, specifically, certain countries in Africa such as Nigeria and Madagascar have relatively lower percentages, though this also reflects relatively higher mortality from other non-injury causes in these countries.

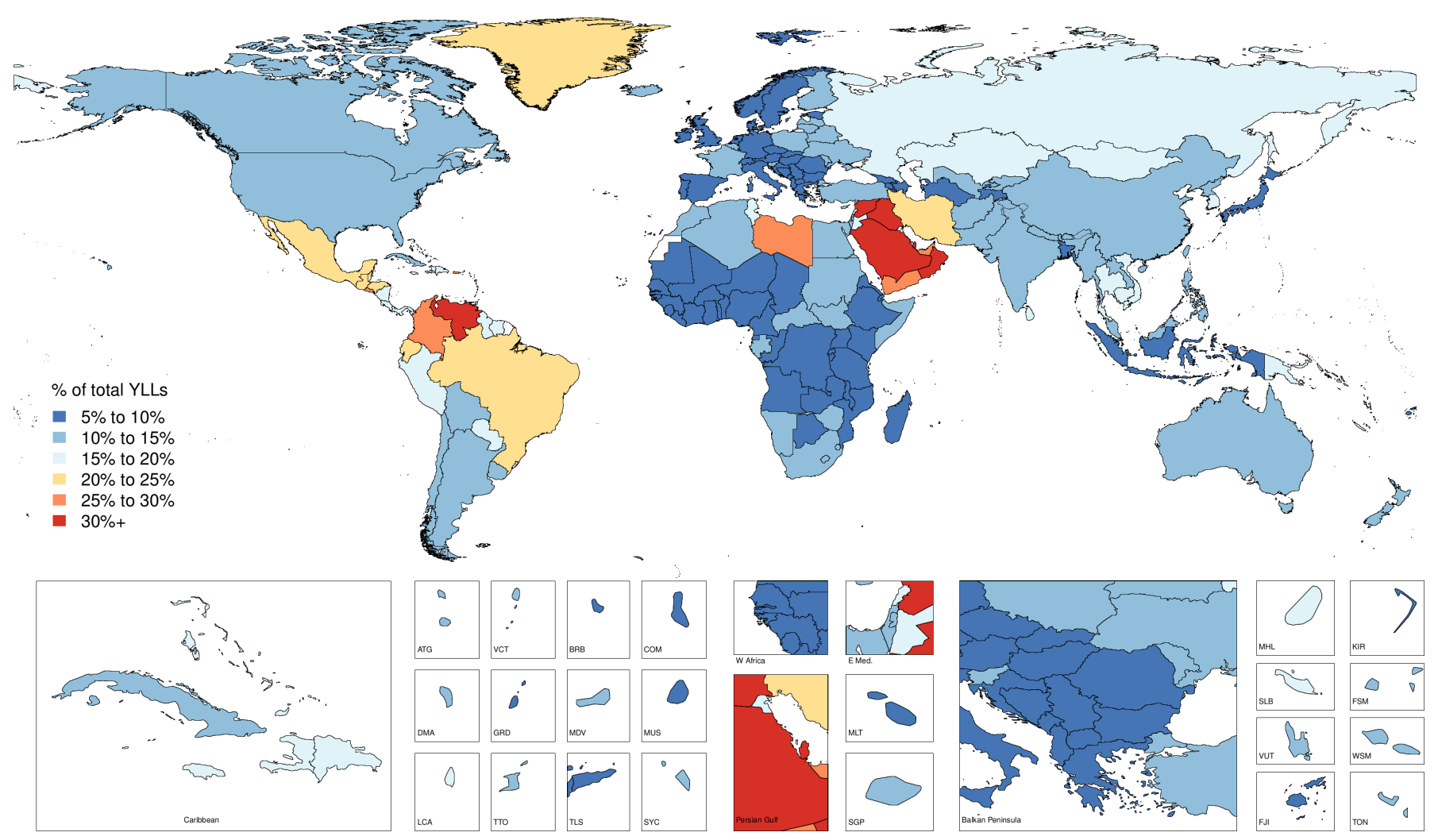

Figure 4 Percentage of YLLs in all ages due to injuries in 2017. YLLs, years of life lost. 


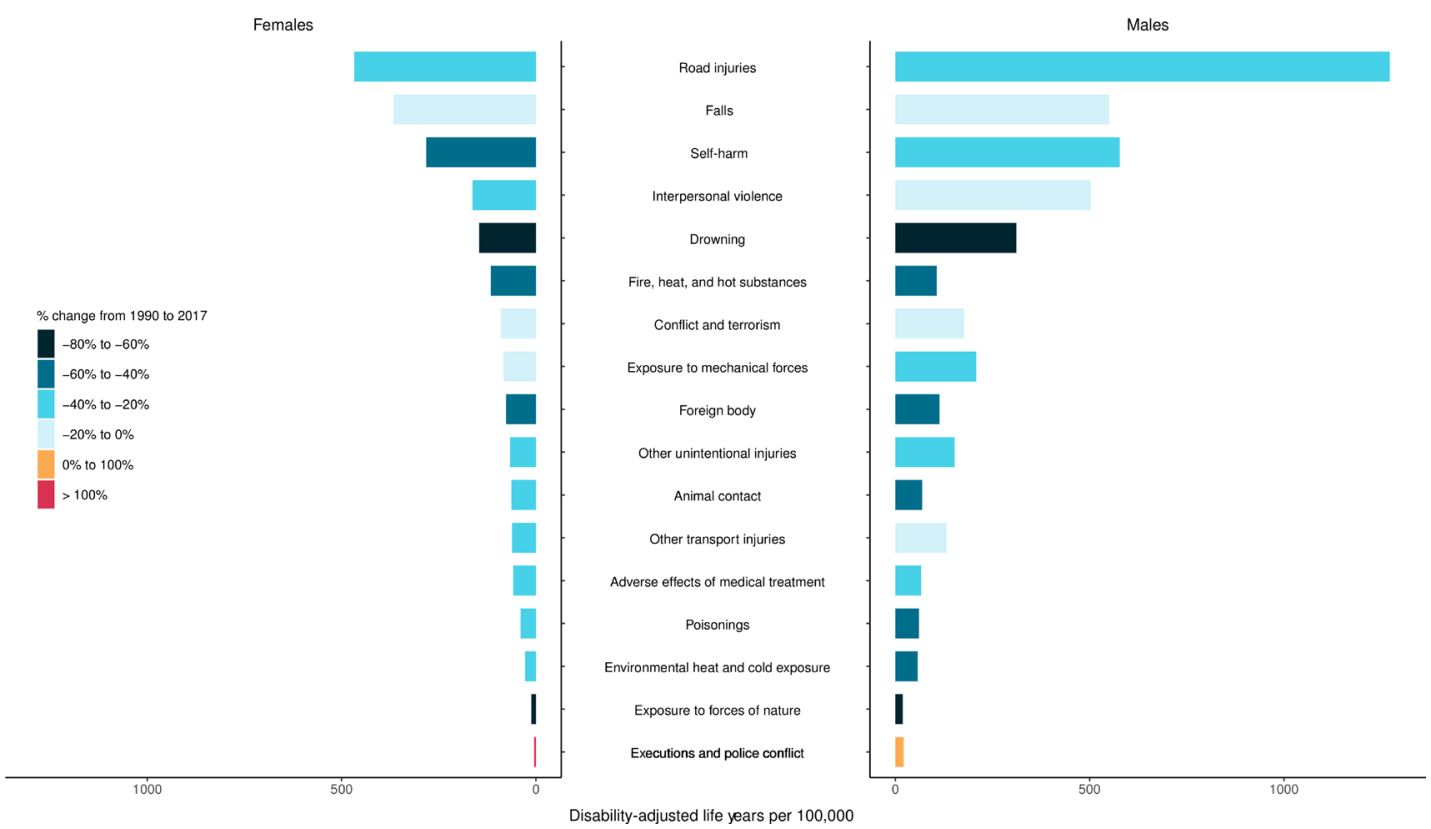

Figure 5 Age-standardised DALY rates by sex for injuries in level 3 of the GBD cause hierarchy in 2017 and percentage change from 1990 to 2017. DALY, disability-adjusted life year; GBD, Global Burden of Disease.

\section{Cause-specific DALY rates by sex}

Figure 5 shows cause-specific DALY rates by sex for 17 injuries in 2017 as well as percentage change from 1990 to 2017 by cause and sex. The black and dark blue bars show causes with greater relative improvement over the time period of this study, while lighter blue, orange and red show injuries that have had lesser improvements, no improvements or increasing burden over time.

In 2017, men experienced higher age-standardised DALY rates than women for all injuries except fire, heat and hot substances. The most marked differences, where DALY rates for men are more than double those of women, can be seen in self-harm, interpersonal violence, road injuries, other transport injuries, exposure to mechanical forces, environmental heat and cold exposure, and executions and police violence. Road injuries (1272 (1209 to 1331) per 100 000), self-harm (577 (525 to 604)) and falls (550 (462 to 653)) were the causes with the highest DALY rates for men in 2017. Women had the highest DALY rates due to the same injuries, but at a lesser magnitude, with rates of 467 (432 to 502) per 100000 for road injuries, 367 (304 to 442) for falls and 282 (268 to 293) for self-harm.

The causes with the largest decreases in DALY rates for men from 1990 to 2017 were exposure to forces of nature $(72.4 \%$ $(63.8 \%$ to $79.1 \%))$, drowning $(62.7 \%(58.8 \%$ to $65.4 \%))$ and fire, heat and hot substances $(43.6 \%(26.4 \%$ to $49.9 \%))$. For women, exposure to forces of nature $(72.8 \%(63.8 \%$ to $79.6 \%)$ ), drowning $(65.8 \%(58.6 \%$ to $69.2 \%))$ and self-harm $(50.8 \%$ $(48.2 \%$ to $55.9 \%)$ ) had the largest decreases in DALY rates. The only increases in DALY rates were seen in executions and police conflict for both women $(298.0 \%(257.1 \%$ to $389.0 \%))$ and men $(46.4 \%$ (31.2\% to $173.0 \%))$.

\section{Comparative regional DALY rates in 2017}

Figure 6 shows a heatmap of the number of standard deviations (SD) above or below the mean of a row (ie, a Z-score) of age-standardised DALY rates for select injuries by GBD region in 2017. For example, the figure shows that the rate of agestandardised DALYs in Eastern Europe is approximately three SD higher than the across mean age-standardised DALY rates of environmental heat and cold exposure across all regions.
Poisonings is also a cause with an age-standardised DALY rate that is approximately three SD higher than in other regions. Positive deviance is seen in high-income Asia Pacific for road injuries, where age-standardised DALYs are one SD lower than the mean across regions. Conversely, Central sub-Saharan Africa has age-standardised DALY rates that are two SD higher than the mean across regions. This figure also demonstrates how certain causes have relatively less variation across regions, for example, most regions do not deviate from the mean age-standardised DALY rates across regions for exposure to forces of nature, with the exception of the Caribbean, which had an age-standardised DALY rate that was approximately four SD above the mean across regions in 2017. Oceania and Eastern Europe stand out as having higher DALY rates for select injuries than other regions, while East Asia, high-income Asia Pacific, high-income North America, Western Europe and Southern Latin America experienced less than average burden of injuries in 2017.

\section{DISCUSSION}

Measuring, understanding and acting on the global burden of injuries should be considered a foundational component of population health research. While this study has reviewed injury burden trends from GBD 2017, it is also evident that these trends are sufficiently different by injury type and geography that it becomes difficult to succinctly generalise the findings in this study. Nevertheless, this study reveals themes and principles germane to the state of global injury burden in 2017 that are relevant to injury burden and prevention research.

First, it should be recognised that despite global population growth with increases in injury cases and deaths, agestandardised death rates from injuries declined from 1990 to 2017. More research into successful improvements for specific injuries in specific countries should be more investigated to help guide efforts towards future improvements. In general terms, the reduction in injury mortality likely represent the combined effects of improvements in healthcare systems, investments in injury prevention programmes and, in certain circumstances, safety improvement such as vehicle safety testing, helmet, seatbelt 


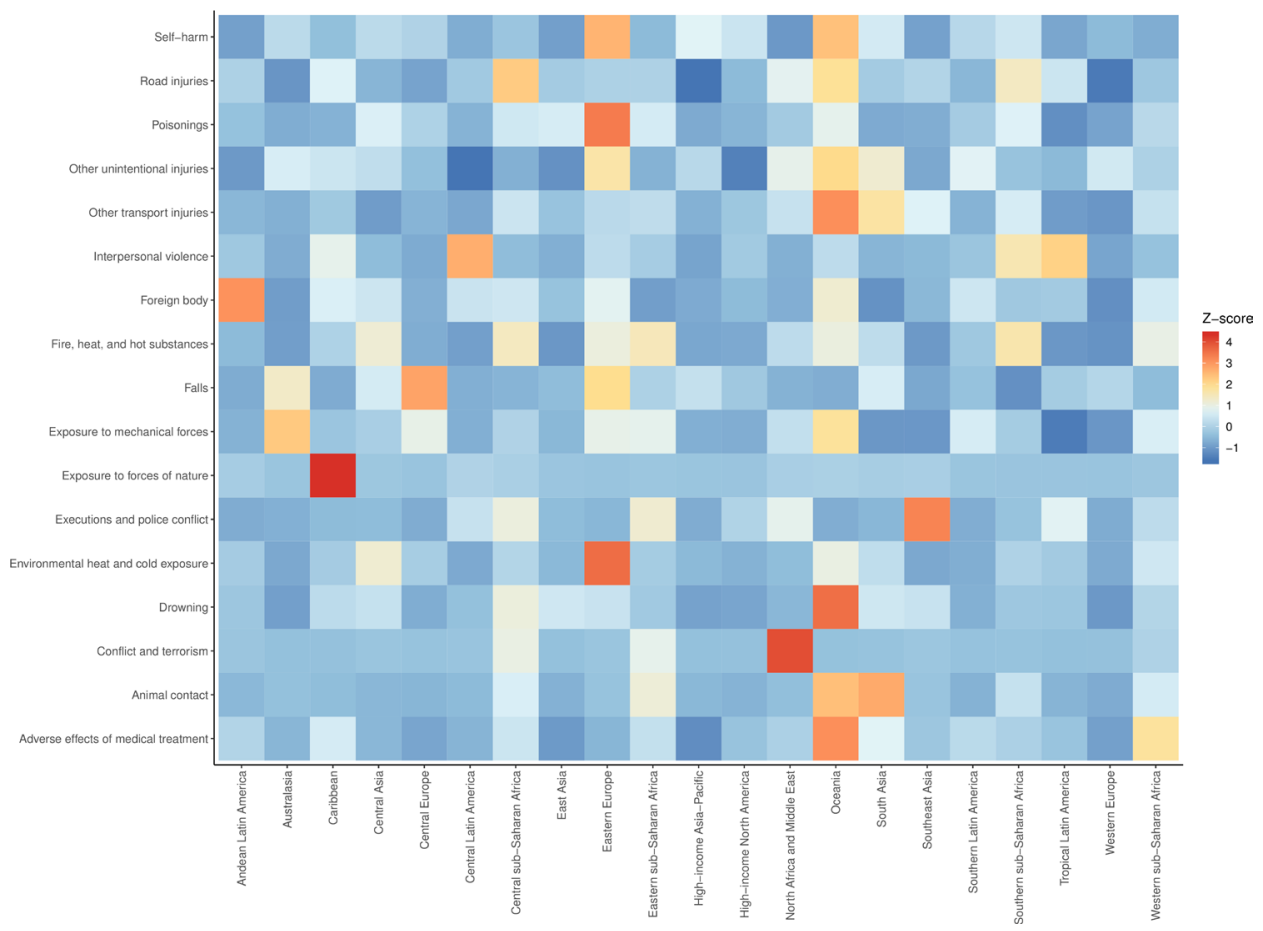

Figure 6 Heatmap showing the Z-score of age-standardised mean DALY rates for select injuries by GBD region in 2017. GBD, Global Burden of Disease.

and drinking and driving laws. While burden trends across all diseases and injuries vary by geography and time, these improvements in injury burden are generally consistent with reporting of communicable and non-communicable disease trends reported in GBD 2017.

Despite improvements in terms of rates, however, it is important to consider the impact of absolute injury burden in younger and adult ages on the social capital and workforce in

\section{What is already known on the subject}

- Injury burden globally varies across many dimensions but remains as an important component of global health loss. Regular updates in injury burden measurement are critical.

- Injuries can be largely preventable, but prevention efforts must be guided by up-to-date estimates of injury burden that can be used on an age-specific, sex-specific, year-specific, location-specific and injury-specific basis.

\section{What this study adds}

- This study incorporates updated data and methods that were used in Global Burden of Disease 2017 with updated burden estimates for the year 2017, as well as newly available results in terms of nature of injury.

- Global age-standardised mortality and disability-adjusted life years decreased between 1990 and 2017. Decreases in agestandardised incidence were not statistically significant.

- Trends over time vary depending on the specific injury, sex and location.

- Injury burden in a population can be radically affected by war, civil conflict and natural disasters. a country. Second, in reviewing temporal trends in figure 2, it becomes evident that war and conflict and environmental disasters can cause profound increases in deaths over a short period of time. This unfortunate and tragic reality should be made more broadly visible as issues such as war, conflict and climate change continue to threaten the populations of the 21st century. Third, sex differentials in the burden of different injury types are large, with men experiencing significantly higher burden from the four leading causes of injury DALYs in 2017. Preventive research and focused interventions into why this is occurring in road injuries, falls, self-harm, interpersonal violence and drowning is critical. It is also critical to address injuries such as fire, heat and hot substance and sexual violence where females experience greater burden and to better understand the factors that drive sex differences. As a fourth theme, we observed that there are cases of both positive and negative deviance from cross-region trends for each injury, as shown in figure 6, which appear to occur even outside of expected differences by income group. For example, understanding why high-income Asia Pacific and Western Europe are performing better than high-income North America in road injury burden could help improve road injury burden even in this higher income setting.

Beyond these four themes, there are evidently a great deal of nuances and specific outcomes to measure and understand in future injury research. While every cause of health loss in a population is important to measure and understand, injuries are unique in that understanding burden requires investigation of an array of circumstances such as infrastructure, the built environment, rates of interpersonal violence in a population and individual behaviours such as alcohol intoxication or drug use. The findings in this paper also demonstrate how it is critical to measure and understand the spectrum of health loss due to injuries ranging from relatively silent injuries to injuries that profoundly affect functional status. An incident as elemental as a trip and fall can lead to profoundly disabling health consequences 
such as spinal cord injury, which can have lifelong disability. The disability caused by shorter term injuries, such as an arm fracture, in addition to causing suffering and disability, can cause loss of human capital. ${ }^{30}$ While this study focused more on the causes of injury as defined in the GBD cause hierarchy, future GBD studies should focus also on depicting the distribution of nature of injury results to better understand how these types of disability affect an individual's functional status. Such analyses become increasingly meaningful as research emerges on, for example, the increased risk of dementia that traumatic brain injury patients may experience. ${ }^{31}$ The findings in this paper also demonstrate how measuring injury burden necessitates review of the population factors that affect injury risk. For example, an event as disastrous as an earthquake may have radically different impacts on a population depending on infrastructure and access to care resources. Understanding how populations can protect themselves against future, unanticipated catastrophe could lead to averted death and disability in the future. As was shown in figure 2, catastrophic events both in terms of natural disasters and war and conflict can significantly add to the death and disability experienced by a population in a short period of time.

The geographical trends shown in this paper are also critical to review and understand by the broader global health community. As shown in figure 6, considerable heterogeneity exists across regions for certain causes. While vehicles were driven in nearly every populated area of earth in 2017, this study shows that different regions of the world have markedly different rates of death and disability resulting from road injuries, underscoring the importance of measuring and understanding the effects of specific factors on injury burden. ${ }^{32}$ It is not necessarily surprising to observe that countries or regions with relatively lower healthcare access and quality, less road safety infrastructure and lower utilisation of vehicles with modern safety standards would have higher rates of road injuries DALYs. The question that extends from this observation, however, is the extent to which burden from this type of injury cause could be avoided were every country to have the safety and prevention factors available in higher income settings. The injury and safety research communities should consider future investigation of counterfactual analyses to better measure and understand the impact that road safety legislation, modernisation of roads and vehicles and improving first response medical care could have on road injury burden, as an example, though parallel examples can be developed for other injury causes as well. This research could help cost-effectiveness analyses and guide investment in safer infrastructure.

These observations converge on a common theme: much of the injury burden may be largely preventable and understanding the success or failure of different prevention efforts should be a prioritised area of health research. Moreover, it is critical for there to be continued engagement across different areas of the world for the purposes of discussing effective and ineffective injury prevention strategies. Dialogue focused on findings across injury prevention efforts via forums such as global safety conferences as well as studies published in research journals should continue to help policy makers and public health planners make strategic investments for preventing future injury burden. ${ }^{33}$ In addition, more research into the cause of injury and resulting bodily injury and environmental and contextual features where injuries occur such type of road in a road injury or fires in factories versus in residences may provide further insight into preventing future injury burden.

Known limitations of injury burden estimation in the GBD framework have been reported previously in peer-reviewed literature. ${ }^{111} 1316$ Generally, identified limitations include data sparsity and correspondingly greater uncertainty in certain geographies, limited geographical coverage of data informing longterm disability estimates and cause-nature relationships, and potential reporting biases for injuries such as self-harm and interpersonal violence. These limitations have been discussed in the aforementioned literature, and this overview study was additionally limited in scope due to the extensive size of the GBD cause hierarchy and location hierarchy. Indeed, over 1400 different cause-nature combinations are available for reporting in the GBD cause hierarchy, and future research would benefit from examining results in the detailed cause hierarchy and across the detailed location, age and sex hierarchy. The GBD Study platform and collaborator network provide a constructive collaborative platform on which future assessments can be conducted and published.

\section{CONCLUSION}

Injury burden is complex but foundational in formulating global health loss. We have identified four broad trends in global injury burden that converge on the principle that injuries should be considered largely preventable but that detailed burden estimates through recent years are a critical global resource to inform meaningful policy. It will be important accurate measurement to continue into the future to guide injury prevention policy.

\section{Author affiliations}

${ }^{1}$ Institute for Health Metrics and Evaluation, University of Washington, Seattle, WA USA

${ }^{2}$ Department of Neurology, Cairo University, Cairo, Egypt

${ }^{3}$ Department of Parasitology and Mycology, Jahrom University of Medical Sciences, Jahrom, Iran

${ }^{4}$ Neuroscience Research Center, Isfahan University of Medical Sciences, Isfahan, Iran ${ }^{5}$ Department of Public Health, Ministry of Health, Riyadh, Saudi Arabia

${ }^{6}$ Department of Orthopaedic Surgery, University of Southern California, Los Angeles,

CA, USA

${ }^{7}$ Department of Public Health, Debre Berhan University, Debre Berhan, Ethiopia

${ }^{8}$ Cardiovascular Medicine, Ain Shams University, Abbasia, Egypt

${ }^{9}$ Department of Medicine, University College Hospital, Ibadan, Ibadan, Nigeria

${ }^{10}$ School of Medicine Center for Politics, Population and Health Research, National

Autonomous University of Mexico, Mexico City, Mexico

"Department of Epidemiology and Health Statistics, Southeast University Nanjing, Nanjing, China

${ }^{12}$ Department of Microbiology, Hazara University Mansehra, Mansehra, Pakistan

${ }^{13}$ James P Grant School of Public Health, BRAC University, Dhaka, Bangladesh

${ }^{14}$ Health Systems and Population Studies Division, International Centre for Diarrhoeal

Disease Research, Bangladesh, Dhaka, Bangladesh

${ }^{15}$ Department of Epidemiology, Jimma University, Jimma, Ethiopia

${ }^{16}$ Higher National School of Veterinary Medicine, Algiers, Algeria

${ }^{17}$ Evidence Based Practice Center, Mayo Clinic Foundation for Medical Education and Research, Rochester, MN, USA

${ }^{18}$ School of Health Sciences, Madda Walabu University, Bale Goba, Ethiopia

${ }^{19}$ Department of Computer Sciences, Imam Abdulrehman Bin Faisal University,

Dammam, Saudi Arabia

${ }^{20}$ Department of Nursing, Debre Markos University, Debre Markos, Ethiopia

${ }^{21}$ Department of Pharmacy, Adigrat University, Adigrat, Ethiopia

${ }^{22}$ Department of Population Health Research, King Abdullah International Medical

Research Center, Riyadh, Saudi Arabia

${ }^{23}$ Faculty of Health Sciences - Health Management and Policy, American University of Beirut, Beirut, Lebanon

${ }^{24}$ British Columbia Injury Research Prevention Unit, British Columbia Children's Hospital Research Institute, Vancouver, BC, Canada

${ }^{25}$ Medical Technical Institute, Erbil Polytechnic University, Erbil, Iraq

${ }^{26}$ Faculty of Pharmacy, Ishik University, Erbil, Iraq

${ }^{27}$ Department of Information Systems, College of Economics and Political Science, Sultan Qaboos University, Muscat, Oman

${ }^{28}$ School of Health Management and Information Sciences, Department of Health Services Management, Iran University of Medical Sciences, Tehran, Iran

${ }^{29}$ Department of Health Care Management and Economics, Urmia University of

Medical Science, Urmia, Iran

${ }^{30}$ Health Management and Economics Research Center, Iran University of Medical Sciences, Tehran, Iran 
${ }^{31}$ Health Economics Department, Iran University of Medical Sciences, Tehran, Iran

${ }^{32}$ Department of Health Policy and Management, Kuwait University, Safat, Kuwait

${ }^{33}$ International Centre for Casemix and Clinical Coding, National University of Malaysia, Bandar Tun Razak, Malaysia

${ }^{34}$ Department of Epidemiology, Arak University of Medical Sciences, Arak, Iran

${ }^{35}$ Physiotherapy Department, The University of Jordan, Amman, Jordan

${ }^{36}$ King Saud University, Riyadh, Saudi Arabia

${ }^{37}$ Clinical Practice Guidelines Unit, King Saud University, Riyadh, Saudi Arabia

${ }^{38}$ Alexandria Center for Evidence-Based Clinical Practice Guidelines, Alexandria University, Alexandria, Egypt

${ }^{39}$ Health Services Management Department, Arak University of Medical Sciences, Arak, Iran

${ }^{40}$ Department of Epidemiology and Biostatistics, University of the Philippines Manila, Manila, Philippines

${ }^{41}$ Online Programs for Applied Learning, Johns Hopkins University, Baltimore, MD, USA

${ }^{42}$ Carol Davila University of Medicine and Pharmacy, Bucharest, Romania

${ }^{43}$ Department of Epidemiology and Biostatistics, Health Promotion Research Center, Zahedan, Iran

${ }^{44}$ Department of Health Policy and Administration, University of the Philippines Manila, Manila, Philippines

${ }^{45}$ Department of Applied Social Sciences, Hong Kong Polytechnic University, Hong Kong, China

${ }^{46}$ Department of Sociology and Social Work, Kwame Nkrumah University of Science and Technology, Kumasi, Ghana

${ }^{47}$ Center for International Health, Ludwig Maximilians University, Munich, Germany

${ }^{48}$ Social Determinants of Health Research Center, Birjand University of Medical

Sciences, Birjand, Iran

${ }^{49}$ Department of Health Promotion and Education, Tehran University of Medical Sciences, Tehran, Iran

${ }^{50}$ School of Health Sciences, Birmingham City University, Birmingham, UK

${ }^{51}$ Regional Centre for the Analysis of Data on Occupational and Work-related Injuries and Diseases, Local Health Unit Tuscany Centre, Florence, Italy

${ }^{52}$ School of Science and Health, Western Sydney University, Sydney, New South Wales, Australia

${ }^{53}$ Oral Health Services, Sydney Local Health District, Sydney, New South Wales, Australia

${ }^{54}$ Department of Plastic Surgery, University of Texas, Houston, TX, USA

${ }^{55}$ Department of Microbiology, Hamedan University of Medical Sciences, Azad Tabriz University, Iran

${ }^{56}$ Department of Nursing, Wolaita Sodo University, Wolaita Sodo, Ethiopia

${ }^{57}$ The Judith Lumley Centre, La Trobe University, Melbourne, VIC, Australia

${ }^{58} \mathrm{General}$ Office for Research and Technological Transfer, Peruvian National Institute of Health, Lima, Peru

${ }^{59}$ School of Public Health, Curtin University, Perth, WA, Australia

${ }^{60}$ Department of Health Policy Planning and Management, University of Health and Allied Sciences, Ho, Ghana

${ }^{61}$ Department of Environmental Health Engineering, Hamadan University of Medical

Sciences, Hamadan, Iran

${ }^{62}$ Public Health Risk Sciences Division, Public Health Agency of Canada, Toronto,

Ontario, Canada

${ }^{63}$ Department of Nutritional Sciences, University of Toronto, Toronto, Ontario, Canada

${ }^{64}$ Department of Forensic Science, Government Institute of Forensic Science, Nagpur, India

${ }^{65}$ Department of Ophthalmology, University Hospital of Ioannina, Ioannina, Greece

${ }^{66}$ Institute of Molecular Biology \& Biotechnology, Foundation for Research \&

Technology, loannina, Greece

${ }^{67}$ Biochemistry Unit, Universiti Sultan Zainal Abidin, Kuala Terengganu, Malaysia

${ }^{68}$ School of Health Sciences, Univeristi Sultan Zainal Abidin, Kuala Terengganu,

Malaysia

${ }^{69}$ Institute of Health Management Research, Indian Institute of Health Management

Research University, Jaipur, India

${ }^{70}$ Department of Epidemiology, Johns Hopkins University, Baltimore, MD, USA

${ }^{71}$ Health Policy And Management Department, Tehran University of Medical Sciences, Tehran, Iran

${ }^{72}$ Department of Demography, University of Groningen, Groningen, Netherlands

${ }^{73}$ Population Research Centre, Institute for Social and Economic Change, Bengaluru, India

${ }^{74}$ Department of Hypertension, Medical University of Lodz, Lodz, Poland

${ }^{75}$ Polish Mothers' Memorial Hospital Research Institute, Lodz, Poland

${ }^{76}$ School of Health Sciences, Walden University, Minneapolis, MN, USA

${ }^{77}$ Department of Noncommunicable Diseases, Bangladesh University of Health

Sciences (BUHS), Dhaka, Bangladesh

${ }^{78}$ Department of Research, Public Health Perspective Nepal, Pokhara-Lekhnath Metropolitan City, Nepal

${ }^{79}$ School of Psychology, University of Auckland, Auckland, New Zealand

${ }^{80}$ Heidelberg Institute of Global Health (HIGH), Heidelberg University, Heidelberg, Germany
${ }^{81}$ T H Chan School of Public Health, Harvard University, Boston, MA, USA

${ }^{82}$ Department of Industrial Engineering, Pontifical Javeriana University, Bogota,

Colombia

${ }^{83}$ Occupational Health Department, Kermanshah University of Medical Sciences,

Kermanshah, Iran

${ }^{84} \mathrm{Health}$ Human Resources Research Center, Shiraz University of Medical Sciences,

Shiraz, Iran

${ }^{85}$ Department of Public Health, Ambo University, Ambo, Ethiopia

${ }^{86}$ Department of Community Medicine, Gandhi Medical College Bhopal, Bhopal, India

${ }^{87}$ Jazan University, Jazan, Saudi Arabia

${ }^{88}$ Social Determinants of Health Research Center, Lorestan University of Medical

Sciences, Khorramabad, Iran

${ }^{89}$ Institute of Health Sciences, School of Public Health, Wollega University, Nekemte, Ethiopia

${ }^{90}$ Department of Psychiatry, Bahir Dar University, Bahir Dar, Ethiopia

${ }^{91}$ Department of Epidemiology and Psychosocial Reseach, Ramón de la Fuente Muñiz

National Institute of Psychiatry, Mexico City, Mexico

${ }^{92}$ Nuffield Department of Population Health, University of Oxford, Oxford, UK

${ }^{93}$ Department of Internal Medicine, University of São Paulo, São Paulo, Brazil

${ }^{94}$ Department of Nutrition and Dietetics, Mekelle University, Mekelle, Ethiopia

${ }^{95}$ Department of Community Medicine and Family Medicine, All India Institute of

Medical Sciences, Jodhpur, India

${ }^{96}$ Department of Community Medicine, Datta Meghe Institute of Medical Sciences,

Wardha, India

${ }^{97}$ Department of Internal Medicine, University of Massachusetts Medical School,

Springfield, MA, USA

${ }^{98}$ Department of Statistical and Computational Genomics, National Institute of

Biomedical Genomics, Kalyani, India

${ }^{99}$ Department of Statistics, University of Calcutta, Kolkata, India

${ }^{100}$ Institute of Soil and Environmental Sciences, University of Agriculture, Faisalabad, Faisalabad, Pakistan

${ }^{101}$ Social Determinants of Health Research Center, Babol University of Medical

Sciences, Babol, Iran

${ }^{102}$ National Centre for Epidemiology and Population Health, Australian National

University, Canberra, ACT, Australia

${ }^{103}$ Department of Clinical Pharmacy and Pharmacology, University of Dhaka, Dhaka, Bangladesh

${ }^{104}$ Department of Clinical and Experimental Medicine, University of Catania, Catania, Italy

${ }^{105}$ Transport and Road Safety (TARS) Research Department, University of New South

Wales, Sydney, New South Wales, Australia

${ }^{106}$ Institute of Epidemiology, Comenius University, Bratislava, Slovakia

${ }^{107}$ Department of Epidemiology and Evidence Based Medicine, I M Sechenov First

Moscow State Medical University, Moscow, Russia

${ }^{108}$ Research Department, Golden Community, Kathmandu, Nepal

${ }^{109} \mathrm{Centre}$ for Population Health Sciences, Nanyang Technological University,

Singapore

${ }^{110} \mathrm{Global}$ eHealth Unit, Imperial College London, London, UK

${ }^{111}$ Department of Population and Health, Metropolitan Autonomous University,

Mexico City, Mexico

${ }^{112}$ Research Unit on Applied Molecular Biosciences (UCIBIO), University of Porto, Porto, Portugal

${ }^{113}$ Department of Psychiatry, University of São Paulo, São Paulo, Brazil

${ }^{114}$ Colombian National Health Observatory, National Institute of Health, Bogota,

Colombia

${ }^{115}$ Epidemiology and Public Health Evaluation Group, National University of Colombia, Bogota, Colombia

${ }^{116}$ Primary Care Services Area, Central Health Directorate, Region Friuli Venezia Giulia, Trieste, Italy

${ }^{117}$ Department of Medicine (DAME), University of Udine, Udine, Italy

${ }^{118}$ National School of Public Health, Carlos III Health Institute, Madrid, Spain

${ }^{119}$ Clinical Epidemiology Program, Ottawa Hospital Research Institute, Ottawa, ON, Canada

${ }^{120}$ Mary MacKillop Institute for Health Research, Australian Catholic University, Melbourne, VIC, Australia

${ }^{121}$ School of Public Health, University of Hong Kong, Hong Kong, China

${ }^{122}$ Institute of Applied Health Research, University of Birmingham, Birmingham, UK

${ }^{123}$ Department of Gynecology and Obstetrics, University of Gondar, Gondar, Ethiopia

${ }^{124}$ Department of Public Health, Texila American University, Georgetown, Guyana

${ }^{125}$ Department of Medicine, University of Toronto, Toronto, Canada

${ }^{126}$ 2nd Department of Ophthalmology, University of Athens, Haidari, Greece

${ }^{127}$ Ophthalmology Private Practice Office, Independent Consultant, Athens, Greece

${ }^{128}$ Department of Pediatrics, Harvard University, Boston, MA, USA

${ }^{129}$ Department of Neonatology, Beth Israel Deaconess Medical center, Boston, MA, USA

${ }^{130}$ Department of Surgery, Division of Plastic and Reconstructive Surgery, University of Washington, Seattle, WA, USA 
${ }^{131}$ Maternal and Child Health Division, International Centre for Diarrhoeal Disease Research, Bangladesh, Dhaka, Bangladesh

${ }^{132}$ Department of Epidemiology and Biostatistics, University of South Carolina, Columbia, SC, USA

${ }^{133}$ Faculty of Biology, Hanoi National University of Education, Hanoi, Vietnam

${ }^{134}$ Research School of Population Health, Australian National University, Action, ACT, Australia

${ }^{135}$ Department of Dermatology, Carol Davila University of Medicine and Pharmacy, Bucharest, Romania

${ }^{136}$ 2nd Department of Dermatology, Colentina Clinical Hospital, Bucharest, Romania

${ }^{137}$ Department of Dermatology, Case Western Reserve University, Cleveland, $\mathrm{OH}$, USA

${ }^{138}$ Department of Dermatology, University of Milan, Milan, Italy

${ }^{139}$ Toxoplasmosis Research Center, Mazandaran University of Medical Sciences, Sari, Iran

${ }^{140}$ Population and Development, Facultad Latinoamericana de Ciencias Sociales Mexico, Mexico City, Mexico

${ }^{141}$ Department of Medical Laboratory Sciences, Bahir Dar University, Bahir Dar, Ethiopia

${ }^{142}$ Department of Nursing, Woldia University, Woldia, Ethiopia

${ }^{143}$ School of Nursing, Jimma University, Jimma, Ethiopia

${ }^{144}$ School of Pharmacy, Aksum University, Aksum, Ethiopia

${ }^{145}$ Addis Ababa University, Addis Ababa, Ethiopia

${ }^{146}$ Immunology Research Center, Tabriz University of Medical Sciences, , Iran

${ }^{147}$ Department of Global Health and Infection, Brighton and Sussex Medical School, Brighton, UK

${ }^{148}$ School of Public Health, Addis Ababa University, Addis Ababa, Ethiopia

${ }^{149}$ Division of Cardiology, Atlanta Veterans Affairs Medical Center, Decatur, GA, USA

${ }^{150}$ Department of Epidemiology, Shiraz University of Medical Sciences, Shiraz, Iran

${ }^{151}$ Faculty of Pharmacy, University of Porto, Porto, Portugal

${ }^{152}$ Tehran University of Medical Sciences, Tehran, Iran

${ }^{153}$ School of Health and Biomedical Sciences, Royal Melbourne Institute of

Technology University, Bundoora, VIC, Australia

${ }^{154}$ Sydney School of Public Health, University of Sydney, Sydney, NSW, Australia

${ }^{155}$ Faculty of Medicine, University of Belgrade, Belgrade, Serbia

${ }^{156}$ Public Health Department, Hawassa University, Hawassa, Ethiopia

${ }^{157}$ Curtin University, Perth, WA, Australia

${ }^{158}$ Department of Global Health and Social Medicine, Harvard University, Boston, MA, USA

${ }^{159}$ Department of Social Services, Tufts Medical Center, Boston, MA, USA

${ }^{160}$ Department of Pharmacology and Toxicology, Maragheh University of Medical

Sciences, Maragheh, Iran

${ }^{161}$ Department of Pharmacology and Toxicology, Tabriz University of Medical Sciences, Tabriz, Iran

${ }^{162}$ National Institute for Health Researches, Tehran University of Medical Sciences, Tehran, Iran

${ }^{163}$ Department of Clinical Pathology, Mansoura University, Mansoura, Egypt

${ }^{164}$ Department of Statistics, Debre Markos University, Debre Markos, Ethiopia

${ }^{165}$ Department of Public Health Sciences, Karolinska Institutet, Stockholm, Sweden

${ }^{166}$ World Health Programme, Université du Québec en Abitibi-Témiscamingue, Rouyn-

Noranda, QC, Canada

${ }^{167}$ Department of Pathology, Stavanger University Hospital, Stavanger, Norway

${ }^{168}$ Norwegian Institute of Public Health, Oslo, Norway

${ }^{169}$ Ophthalmic Epidemiology Research Center, Shahroud University of Medical Sciences, Shahroud, Iran

${ }^{170}$ Department of Midwifery, Wolkite University, Wolkite, Ethiopia

${ }^{171}$ Multiple Sclerosis Research Center, Tehran University of Medical Sciences, Tehran, Iran

${ }^{172}$ Biology Department, Salahaddin University-Erbil, Erbil, Iraq

${ }^{173}$ Biology and Biotechnolaniogy"L Spallanzani", University of Pavia, Pavia, Italy

${ }^{174}$ Department of Psychology, Federal University of Sergipe, Sao Cristovao, Brazi

${ }^{175}$ Non-communicable Diseases Research Center, Tehran University of Medical Sciences, Tehran, Iran

${ }^{176}$ Pharmaceutical Nanotechnology, Tehran University of Medical Sciences, Tehran, Iran

${ }^{177}$ Department of Psychiatry, Addis Ababa University, Addis Ababa, Ethiopia

${ }^{178}$ Nursing Department, Hawassa University, Hawassa, Ethiopia

${ }^{179}$ Department of Neurobiology, Karolinska Institutet, Stockholm, Sweden

${ }^{180}$ Division of Neurology, University of Ottawa, Ottawa, ON, Canada

${ }^{181}$ REQUIMTE/LAQV, University of Porto, Porto, Portugal

${ }^{182}$ Research Centre on Public Health (CESP), University of Milan Bicocca, Monza, Italy

${ }^{183}$ Department of Health Education \& Behavioral Sciences, Jimma University, Jimma,

Ethiopia

${ }^{184}$ Psychiatry Department, Kaiser Permanente, Fontana, CA, USA

${ }^{185}$ School of Health Sciences, A T Still University, Mesa, AZ, USA

${ }^{186}$ Department of Population Medicine and Health Services Research, Bielefeld University, Bielefeld, Germany

${ }^{187}$ Department of Child Dental Health, Obafemi Awolowo University, Ile-Ife, Nigeria
${ }^{188}$ Abadan School of Medical Sciences, Abadan University of Medical Sciences, Abadan, Iran

${ }^{189}$ Department of Family Medicine and Primary Care, University of the Witwatersrand, Johannesburg, South Africa

${ }^{190}$ College of Public Health, Medical and Veterinary Science, James Cook University, Douglas, QLD, Australia

${ }^{191}$ Royal Life Saving Society, Sydney, NSW, Australia

${ }^{192}$ Department of Dermatology, Kobe University, Kobe, Japan

${ }^{193}$ Gene Expression \& Regulation Program, The Wistar Institute, Philadelphia, PA, USA

${ }^{194}$ Public Health Department, Madda Walabu University, Bale-Robe, Ethiopia

${ }^{195}$ School of Pharmacy, Mekelle University, Mekelle, Ethiopia

${ }^{196}$ Department of Nursing and Midwifery, Addis Ababa University, Addis Ababa,

Ethiopia

${ }^{197}$ Department of Nursing, Aksum University, Aksum, Ethiopia

${ }^{198}$ Department of Nursing, Mekelle University, Mekelle, Ethiopia

${ }^{199}$ School of Public Health, Haramaya University, Harar, Ethiopia

${ }^{200}$ Bahir Dar University, Bahir Dar, Ethiopia

${ }^{201}$ Haramaya University, Dire Dawa, Ethiopia

${ }^{202}$ Department of Pharmacy, Wollo University, Dessie, Ethiopia

${ }^{203}$ Department of Medical Surgery, Tabriz University of Medical Sciences, Tabriz, Iran

${ }^{204}$ Occupational Health Department, Arak University of Medical Sciences, Arak, Iran

${ }^{205}$ Department of Health Services Management, Iran University of Medical Sciences, Tehran, Iran

${ }^{206}$ Science and Research Branch, Islamic Azad University, Tehran, Iran

${ }^{207}$ Young Researchers and Elite Club, Rasht Branch, Islamic Azad University, Rasht, Iran

${ }^{208}$ Adelaide Medical School, University of Adelaide, Adelaide, SA, Australia

${ }^{209}$ Center for Clinical and Epidemiological Research, University of São Paulo, Sao

Paulo, Brazi

${ }^{210}$ Department of Dermatology, Boston University, Boston, MA, USA

${ }^{211}$ Institute of Public Health, United Arab Emirates University, Al Ain, United Arab

Emirates

${ }^{212}$ Technical College of Health, Sulaimani Polytechnic University, Sulaimani, Irac

${ }^{213}$ Instituto de Patologia Tropical e Saúde Pública, Federal University of Goias,

Goiânia, Brazil

${ }^{214}$ School of Public Health and Preventive Medicine, Monash University, Melbourne,

VIC, Australia

${ }^{215}$ Department of Epidemiology and Biostatistics, Zhengzhou University, Zhengzhou,

China

${ }^{216}$ Non-Communicable Diseases (NCD), World Health Organization (WHO), New

Delhi, India

${ }^{217}$ Department of Public Health, Erasmus University Medical Center, Rotterdam,

Netherlands

${ }^{218}$ Department of Radiology and Radiological Sciences, Johns Hopkins University,

Baltimore, MD, USA

${ }^{219}$ School of Medicine, Tehran University of Medical Sciences, Tehran, Iran

${ }^{220}$ Institute for Global Health, University College London, London, UK

${ }^{221} \mathrm{Global}$ and Community Mental Health Research Group, University of Macau,

Macao, China

${ }^{222}$ Department of Family and Community Medicine, Arabian Gulf University,

Manama, Bahrain

${ }^{223}$ School of Health and Environmental Studies, Hamdan Bin Mohammed Smart

University, Dubai, United Arab Emirates

${ }^{224}$ Biomedical Research Networking Center for Mental Health Network (CiberSAM),

Madrid, Spain

${ }^{225}$ Research and Development Unit, San Juan de Dios Sanitary Park, Sant Boi de Llobregat, Spain

${ }^{226}$ Institute for Social Science Research, The University of Queensland, Indooroopilly, QLD, Australia

${ }^{227}$ Department of Microbiology, Maragheh University of Medical Sciences, Maragheh, Iran

${ }^{228}$ Department of Microbiology, Tehran University of Medical Sciences, Tehran, Iran

${ }^{229}$ Gastrointestinal and Liver Disease Research Center, Guilan University of Medical

Sciences, Rasht, Iran

${ }^{230}$ School of Nursing and Midwifery, Tabriz University of Medical Sciences, Tabriz, Iran

${ }^{231}$ Independent Consultant, Tabriz, Iran

${ }^{232}$ Department of Public Health, Mizan-Tepi University, Tepi, Ethiopia

${ }^{233}$ Unit of Epidemiology and Social Medicine, University Hospital Antwerp, Wilrijk

Belgium

${ }^{234}$ Department of Clinical Sciences, Karolinska University Hospital, Stockholm,

Sweden

${ }^{235}$ Institute of Pharmaceutical Sciences, University of Veterinary and Animal Sciences,

Lahore, Pakistan

${ }^{236}$ Department of Pharmacy Administration and Clinical Pharmacy, Xian Jiaotong

University, Xian, China

${ }^{237}$ Medical Biology Research Center, Kermanshah University of Medical Sciences, Kermanshah, Iran

${ }^{238}$ Research Coordination, AC Environments Foundation, Cuernavaca, Mexico 
${ }^{239} \mathrm{CISS}$, National Institute of Public Health, Cuernavaca, Mexico

${ }^{240}$ Department of Urban Planning and Design, University of Hong Kong, Hong Kong, China

${ }^{241}$ Center of Excellence in Behavioral Medicine, Nguyen Tat Thanh University, Ho Chi Minh City, Vietnam

${ }^{242}$ Department of Pediatrics, Dell Medical School, University of Texas Austin, Austin, TX, USA

${ }^{243}$ Kasturba Medical College, Manipal Academy of Higher Education, Manipal, India

${ }^{244}$ Department of Pharmacology and Therapeutics, Dhaka Medical College, Dhaka, Bangladesh

${ }^{245}$ Department of Pharmacology, Bangladesh Industrial Gases Limited, Tangail, Bangladesh

${ }^{246}$ Department of Computer Engineering, Islamic Azad University, Tehran, Iran

${ }^{247}$ Computer Science Department, University of Human Development, Sulaymaniyah, Iraq

${ }^{248}$ Department of Legal Medicine and Bioethics, Carol Davila University of Medicine and Pharmacy, Bucharest, Romania

${ }^{249}$ Clinical Legal Medicine Department, National Institute of Legal Medicine Mina Minovici, Bucharest, Romania

${ }^{250}$ Department of Epidemiology and Health Statistics, Central South University, Changsha, China

${ }^{251}$ Department of Health Promotion and Education, University of Ibadan, Ibadan, Nigeria

${ }^{252}$ Department of Community Medicine, University of Ibadan, Ibadan, Nigeria

${ }^{253}$ Department of Epidemiology, University of Kragujevac, Kragujevac, Serbia

${ }^{254}$ Department of Family Medicine, Bangalore Baptist Hospital, Bangalore, India

${ }^{255}$ Center for Health Resource and Services Research and Development, National Institute of Health Research \& Development, Jakarta, Indonesia

${ }^{256}$ Research Institute for Endocrine Sciences, Shahid Beheshti University of Medical Sciences, Tehran, Iran

${ }^{257}$ Institute for Physical Activity and Nutrition, Deakin University, Burwood, VIC, Australia

${ }^{258}$ Sydney Medical School, University of Sydney, Sydney, NSW, Australia

${ }^{259}$ School of Psychology and Public Health, La Trobe University, Bundoora, Melbourne, VIC, Australia

${ }^{260}$ School of Public Health and Community Medicine, University of New South Wales, Sydney, Australia

${ }^{261}$ Department of Global and Community Health, George Mason University, Fairfax, VA, USA

${ }^{262}$ Faculty of Medicine, Babol University of Medical Sciences, Babol, Iran

${ }^{263}$ School of Management and Medical Education, Shahid Beheshti University of

Medical Sciences, Tehran, Iran

${ }^{264}$ Safety Promotion and Injury Prevention Research Center, Shahid Beheshti

University of Medical Sciences, Tehran, Iran

${ }^{265}$ Department for Health Care and Public Health, I M Sechenov First Moscow State Medical University, Moscow, Russia

${ }^{266}$ Social Development \& Health Promotion Research Center, Kermanshah University of Medical Sciences, Kermanshah, Iran

${ }^{267}$ Department of Surgery, Virginia Commonwealth University, Richmond, VA, USA

${ }^{268}$ Institute of Medicine, University of Colombo, Colombo, Sri Lanka

${ }^{269}$ Faculty of Graduate Studies, University of Colombo, Colombo, Sri Lanka

${ }^{270}$ Department of Community Medicine, Banaras Hindu University, Varanasi, India

${ }^{271}$ Department of Ophthalmology, Heidelberg University, Mannheim, Germany

${ }^{272}$ Beijing Ophthalmology \& Visual Science Key Laboratory, Beijing Tongren Hospital, Beijing, China

${ }^{273}$ Department of Community Medicine, Kasturba Medical College, Manipal Academy of Higher Education, Mangalore, India

${ }^{274}$ Department of Family Medicine and Public Health, University of Opole, Opole, Poland

${ }^{275}$ School of Health Sciences, Savitribai Phule Pune University, Pune, India

${ }^{276}$ Institute of Family Medicine and Public Health, University of Tartu, Tartu, Estonia

${ }^{277}$ Minimally Invasive Surgery Research Center, Iran University of Medical Sciences, Tehran, Iran

${ }^{278}$ Personal Social Services Research Unit, London School of Economics and Political Science, London, UK

${ }^{279}$ Department of Medical Informatics, Tabriz University of Medical Sciences, Tabriz, Iran

${ }^{280}$ Social Determinants of Health Research Center, Research Institute for Prevention of Non-Communicable Diseases, Qazvin University of Medical Sciences, Qazvin, Iran

${ }^{281}$ Health Services Management Department, Qazvin University of Medical Sciences, Oazvin, Iran

${ }^{282}$ School of Public Health, Department of Health Informatics and Health Innovation, A C S Medical College and Hospital, Mekelle, Ethiopia

${ }^{283}$ Department of Forensic Medicine and Toxicology, All India Institute of Medical Sciences, Jodhpur, India

${ }^{284}$ Department of Epidemiology, Hamadan University of Medical Sciences, Hamadan, Iran
${ }^{285}$ Hematology-Oncology and Stem Cell Transplantation Research Center, Tehran University of Medical Sciences, Tehran, Iran

${ }^{286}$ Pars Advanced and Minimally Invasive Medical Manners Research Center, Iran University of Medical Sciences, Tehran, Iran

${ }^{287}$ Department of Applied Physics, The John Paul II Catholic University of Lublin, Lublin Voivodeship, Poland

${ }^{288}$ Department of Biology and Chemistry, Drohobych Ivan Franko State Pedagogical University, Drohobych, Ukraine

${ }^{289}$ Department of Pharmacy, Jimma University, Jimma, Ethiopia

${ }^{290}$ Open, Distance and eLearning Campus, University of Nairobi, Nairobi, Kenya

${ }^{291}$ Department of Dermatology, Wolaita Sodo University, Wolaita Sodo, Ethiopia

${ }^{292}$ Department of Midwifery, University of Gondar, Gondar, Ethiopia

${ }^{293}$ Department of Public Health, Jordan University of Science and Technology, Irbid, Jordan

${ }^{294}$ Social Determinants of Health Research Center, Ahvaz Jundishapur University of

Medical Sciences, Ahvaz, Iran

${ }^{295}$ School of Food and Agricultural Sciences, University of Management and

Technology, Lahore, Pakistan

${ }^{296}$ Department of Global Health, University of Washington, Seattle, WA, USA

${ }^{297}$ Department of Physiology, Baku State University, Baku, Azerbaijan

${ }^{298}$ Health Care Management, Zahedan University of Medical Sciences, zahedan, Iran

${ }^{299}$ Epidemiology and Biostatistics Department, Health Services Academy, Islamabad, Pakistan

${ }^{300}$ Faculty of Public Health and Tropical Medicine, Jazan University, Jazan, Saudi Arabia

${ }^{301}$ Department of Health Research, Indian Council of Medical Research, New Delhi, India

${ }^{302}$ Centre for Ethics, Jawahar Lal Nehru University, New Delhi, India

${ }^{303}$ Department of Psychiatry, Kermanshah University of Medical Sciences, Kermanshah, Iran

${ }^{304}$ Department of Epidemiology, Kermanshah University of Medical Sciences, Kermanshah, Iran

${ }^{305}$ Nuffield Department of Surgical Sciences, Oxford University Global Surgery Group, University of Oxford, Oxford, UK

${ }^{306}$ Research and Data Solutions, Synotech Consultant, Nairobi, Kenya

${ }^{307}$ Department of Preventive Medicine, Korea University, Seoul, South Korea

${ }^{308}$ Department of Health Sciences, Northeastern University, Boston, MA, USA

${ }^{309}$ School of Medicine, Xiamen University Malaysia, Sepang, Malaysia

${ }^{310}$ School of Health Sciences, Kristiania University College, Oslo, Norway

${ }^{311}$ Department of Nursing and Health Promotion, Oslo Metropolitan University, Oslo, Norway

${ }^{312}$ Neurophysiology Research Center, Hamadan University of Medical Sciences,

Hamadan, Iran

${ }^{313}$ Brain Engineering Research Center, Institute for Research in Fundamental

Sciences, Tehran, Iran

${ }^{314}$ Department of Public Health Dentistry, Deemed University, karad, India

${ }^{315}$ CIBERSAM, San Juan de Dios Sanitary Park, Sant Boi de Llobregat, Spain

${ }^{316}$ Catalan Institution for Research and Advanced Studies (ICREA), Barcelona, Spain

${ }^{317}$ Department of Zoology, University of Oxford, Oxford, UK

${ }^{318}$ Harvard Medical School, Harvard University, Boston, MA, USA

${ }^{319}$ Department of Anthropology, Panjab University, Chandigarh, India

${ }^{320}$ Department of Public Health, Yuksek Ihtisas University, Ankara, Turkey

${ }^{321}$ Department of Public Health, Hacettepe University, Ankara, Turkey

${ }^{322}$ Department of Family and Community Health, University of Health and Allied

Sciences, Ho, Ghana

${ }^{323}$ Department of Psychology and Health Promotion, University of KwaZulu-Natal, Durban, South Africa

${ }^{324}$ Department of Medicine, Brigham and Women's Hospital, Harvard University,

Boston, MA, USA

${ }^{325}$ Public Health Foundation of India, Gurugram, India

${ }^{326}$ Department of Psychiatry, University of Nairobi, Nairobi, Kenya

${ }^{327}$ Institute of Occupational and Environmental Medicine, University of Birmingham, Birmingham, UK

${ }^{328}$ Mechanical and Industrial Engineering, Indian Institute of Technology, Roorkee,

Roorkee, India

${ }^{329}$ Department of Medicine, McMaster University, Hamilton, ON, Canada

${ }^{330} \mathrm{Health}$ and Nutrition Section, United Nations Childrens' Fund (UNICEF), Accra, Ghana

${ }^{331}$ Department of Clinical Medicine and Community Health, University of Milan,

Milano, Italy

${ }^{332}$ Department of Community and Family Medicine, University of Baghdad, Baghdad, Iraq

${ }^{333}$ School of Medicine, Deakin University, Geelong, VIC, Australia

${ }^{334}$ Health Promotion and Chronic Disease Prevention Branch, Public Health Agency of

Canada, Ottawa, ON, Canada

${ }^{335} \mathrm{HelpMeSee,} \mathrm{New} \mathrm{York,} \mathrm{NY,} \mathrm{USA}$

${ }^{336}$ International Relations, Mexican Institute of Ophthalmology, Queretaro, Mexico

${ }^{337}$ Disease Control Department, Ghana Health Service, Accra, Ghana 
${ }^{338}$ Department of Otorhinolaryngology (ENT), Father Muller Medical College, Mangalore, India

${ }^{339}$ Department of Public Health, Maragheh University of Medical Sciences, Maragheh, Iran

${ }^{340}$ Institute of Clinical Physiology, Italian National Research Council, Pisa, Italy

${ }^{341}$ College of Optometry, Nova Southeastern University, Fort Lauderdale, FL, USA

${ }^{342}$ School of Pharmacy, Monash University, Bandar Sunway, Malaysia

${ }^{343}$ School of Pharmacy, Taylor's University Lakeside Campus, Subang Jaya, Malaysia

${ }^{344}$ School of Public Health, Wolaita Sodo University, Wolaita Sodo, Ethiopia

${ }^{345}$ Department of Health Sciences, University of Florence, Florence, Italy

${ }^{346}$ School of Public Health, University of Haifa, Haifa, Israel

${ }^{347}$ Department of Systems, Populations and Leadership, University of Michigan, Ann Arbor, MI, USA

${ }^{348}$ School of Population and Global Health, University of Melbourne, Melbourne, VIC, Australia

${ }^{349}$ Department of Health Metrics Sciences, School of Medicine, University of Washington, Seattle, WA, USA

${ }^{350}$ Department of Medicine, University of São Paulo, Sao Paulo, Brazil

${ }^{351}$ Department of General Surgery, Aintree University Hospital National Health

Service (NHS) Foundation Trust, Liverpool, UK

${ }^{352}$ Department of Surgery, University of Liverpool, Liverpool, UK

${ }^{353}$ Health Data Research UK, Swansea University, Swansea, UK

${ }^{354}$ College of Medicine, Pathology Department, Imam Abdulrahman Bin Faisal University, Dammam, Saudi Arabia

${ }^{355}$ Ophthalmology Department, Aswan Faculty of Medicine, Aswan, Egypt

${ }^{356}$ Institute of Medicine, Tribhuvan University, Kathmandu, Nepal

${ }^{357}$ Department of Public Health, Trnava University, Trnava, Slovakia

${ }^{358}$ Department of Primary Care and Public Health, Imperial College London, London, UK

${ }^{359}$ Public Health Research Department, National Health Institute Colombia, Bogota, Colombia

${ }^{360}$ Faculty of Medicine, El Bosque University, Bogota, Colombia

${ }^{361}$ Health Education and Research Department, SDM College of Medical Sciences \& Hospital, Dharwad, India

${ }^{362}$ Rajiv Gandhi University of Health Sciences, Bangalore, India

${ }^{363}$ Digestive Diseases Research Institute, Tehran University of Medical Sciences,

Tehran, Iran

${ }^{364}$ Non-communicable Diseases Research Center, Shiraz University of Medical

Sciences, Shiraz, Iran

${ }^{365}$ Department of Maternal and Child Nursing and Public Health, Federal University of Minas Gerais, Belo Horizonte, Brazil

${ }^{366}$ Department of Ophthalmology, Iran University of Medical Sciences, Tehran, Iran

${ }^{367}$ Ophthalmology Department, University of Manitoba, Winnipeg, MB, Canada

${ }^{368}$ Department of Surgery, University of Virginia, Charlottesville, VA, USA

${ }^{369}$ Surgery Department, Emergency University Hospital Bucharest, Bucharest, Romania

${ }^{370}$ Department of Psychiatry, National Institute of Mental Health and Neurosciences, Bengaluru, India

${ }^{371}$ Substance Abuse Prevention Research Center, Kermanshah University of Medical

Sciences, Kermanshah, Iran

${ }^{372}$ Department of Epidemiology and Biostatistics, Tehran University of Medical

Sciences, Tehran, Iran

${ }^{373}$ Institute for Social Science Research, The University of Queensland, Brisbane, QLD, Australia

${ }^{374}$ Institute of Bone and Joint Research, University of Sydney, St Leonards, NSW, Australia

${ }^{375}$ Department of Health Sciences, University of York, York, UK

${ }^{376}$ Department of Midwifery-Reproductive Health, Hamadan University of Medical Sciences, Hamadan, Iran

${ }^{377}$ Research Department, The George Institute for Global Health, New Delhi, India

${ }^{378}$ School of Medicine, University of New South Wales, Sydney, NSW, Australia

${ }^{379}$ Department of Public Health, Mekelle University, Mekelle, Ethiopia

${ }^{380}$ Department of Nursing, Arba Minch University, Arba Minch, Ethiopia

${ }^{381}$ Division of Epidemiology and Prevention, Institute of Human Virology, University of Maryland, Baltimore, MD, USA

${ }^{382}$ Peru Country Office, United Nations Population Fund (UNFPA), Lima, Peru

${ }^{383}$ Forensic Medicine Division, Imam Abdulrahman Bin Faisal University, Dammam, Saudi Arabia

${ }^{384}$ College of Health Science, Department of Midwifery, Adigrat University, Adigrat, Ethiopia

${ }^{385}$ Department of Epidemiology and Biostatistics, Haramaya University, Harar, Ethiopia

${ }^{386}$ Breast Surgery Unit, Helsinki University Hospital, Helsinki, Finland

${ }^{387}$ University of Helsinki, Helsinki, Finland

${ }^{388}$ Neurocenter, Helsinki University Hospital, Helsinki, Finland

${ }^{389}$ School of Health Sciences, University of Melbourne, Parkville, VIC, Australia

${ }^{390}$ Clinical Microbiology and Parasitology Unit, ZoraProfozic Polyclinic, Zagreb, Croatia
${ }^{391}$ University Centre Varazdin, University North, Varazdin, Croatia

${ }^{392}$ Center for Innovation in Medical Education, Pomeranian Medical University,

Szczecin, Poland

${ }^{393}$ Department of Propedeutics of Internal Diseases \& Arterial Hypertension,

Pomeranian Medical University, Szczecin, Poland

${ }^{394}$ Pacific Institute for Research \& Evaluation, Calverton, MD, USA

${ }^{395}$ Achutha Menon Centre for Health Science Studies, Sree Chitra Tirunal Institute for

Medical Sciences and Technology, Trivandrum, India

${ }^{396} \mathrm{Global}$ Institute of Public Health (GIPH), Ananthapuri Hospitals and Research

Centre, Trivandrum, India

${ }^{397}$ Department of Statistics and Econometrics, Bucharest University of Economic

Studies, Bucharest, Romania

${ }^{398}$ President's Office, National Institute of Statistics Romania, Bucharest, Romania

${ }^{399}$ Faculty of Internal Medicine, Kyrgyz State Medical Academy, Bishkek, Kyrgyzstan

${ }^{400}$ Department of Atherosclerosis and Coronary Heart Disease, National Center of

Cardiology and Internal Disease, Bishkek, Kyrgyzstan

${ }^{401}$ Heidelberg Institute of Global Health (HIGH), Faculty of Medicine and University

Hospital, Heidelberg University, Heidelberg, Germany

${ }^{402}$ Institute of Addiction Research (ISFF), Frankfurt University of Applied Sciences,

Frankfurt, Germany

${ }^{403}$ Biotechnology Research Center, Tabriz University of Medical Sciences, Tabriz, Iran

${ }^{404}$ Molecular Medicine Research Center, Tabriz University of Medical Sciences, Tabriz, Iran

${ }^{405}$ Health Equity Research Center, Tehran University of Medical Sciences, Tehran, Iran

${ }^{406}$ Internal Medicine Department, King Saud University, Riyadh, Saudi Arabia

${ }^{407}$ Research Center, Salahaddin University, Erbil, Iraq

${ }^{408}$ Ishik University, Erbil, Iraq

${ }^{409}$ Department of Information Technology, University of Human Development,

Sulaymaniyah, Iraq

${ }^{410}$ Department of Biostatistics, Hamadan University of Medical Sciences, Hamadan, Iran

${ }^{411}$ Department of Epidemiology and Biostatistics, Shahrekord University of Medical

Sciences, Shahrekord, Iran

${ }^{412}$ Department of Clinical Biochemistry, Faculty of Medicine, Gonabad University of Medical Sciences, Gonabad, Iran

${ }^{413}$ Department of Nursing, Shahroud University of Medical Sciences, Shahroud, Iran

${ }^{414}$ Health Systems and Policy Research Unit, Ahmadu Bello University, Zaria, Nigeria

${ }^{415}$ Department of Public Health, Samara University, Samara, Ethiopia

${ }^{416}$ Iran National Institute of Health Research, Tehran University of Medical Sciences,

Tehran, Iran

${ }^{417}$ Faculty of Life Sciences and Medicine, King's College London, London, UK

${ }^{418}$ Clinical Epidemiology and Public Health Research Unit, Burlo Garofolo Institute for

Maternal and Child Health, Trieste, Italy

${ }^{419}$ Department of Public Health Medicine, University of KwaZulu-Natal, Durban, South Africa

${ }^{420} \mathrm{Health}$ Sciences Research Center, Mazandaran University of Medical Sciences, Sari, Iran

${ }^{421}$ Research Center for Environmental Determinants of Health, Kermanshah

University of Medical Sciences, Kermanshah, Iran

${ }^{422}$ Social Determinants of Health Research Center, Kurdistan University of Medical

Sciences, Sanandaj, Iran

${ }^{423}$ Department of Epidemiology and Biostatistics, Kurdistan University of Medical

Sciences, Sanandaj, Iran

${ }^{424}$ Preventive Medicine and Public Health Research Center, Iran University of Medical

Sciences, Tehran, Iran

${ }^{425}$ International Laboratory for Air Quality and Health, Queensland University of Technology, Brisbane, QLD, Australia

${ }^{426}$ Gorgas Memorial Institute for Health Studies, Panama City, Panama

${ }^{427}$ Department of Social Medicine, National Center for Child Health and

Development, Setagaya, Japan

${ }^{428}$ Department of Epidemiology and Biostatistics, University of Gondar, Gondar,

Ethiopia

${ }^{429}$ Department of Pediatrics, University of British Columbia, Vancouver, BC, Canada

${ }^{430}$ School of Medical Sciences, Science University of Malaysia, Kubang Kerian, Malaysia

${ }^{431}$ Department of Pediatric Medicine, Nishtar Medical University, Multan, Pakistan

${ }^{432}$ Department of Pediatrics \& Pediatric Pulmonology, Institute of Mother \& Child

Care, Multan, Pakistan

${ }^{433}$ Department of Obstetrics and Gynecology, Ain Shams University, Cairo, Egypt

${ }^{434} \mathrm{~K}$ nowledge Translation and Utilization, Egyptian Center for Evidence Based

Medicine, Egypt

${ }^{435}$ Research and Analytics, Initiative for Financing Health and Human Development,

Chennai, India

${ }^{436}$ Research and Analytics, Bioinsilico Technologies, Chennai, India

${ }^{437}$ Department of Epidemiology, University of Alabama at Birmingham, Birmingham, AL, USA

${ }^{438}$ Laboratory of Public Health Indicators Analysis and Health Digitalization, Moscow Institute of Physics and Technology, Dolgoprudny, Russia 
${ }^{439}$ Experimental Surgery and Oncology Laboratory, Kursk State Medical University of the Ministry of Health of the Russian Federation, Kursk, Russia

${ }^{440}$ Department of Epidemiology \& Biostatistics, Kermanshah University of Medical Sciences, Kermanshah, Iran

${ }^{441}$ Suraj Eye Institute, Nagpur, India

${ }^{442}$ Hospital of the Federal University of Minas Gerais, Federal University of Minas Gerais, Belo Horizonte, Brazil

${ }^{443}$ Mental Health Research Center, Iran University of Medical Sciences, Tehran, Iran

${ }^{444}$ Department of Forensic Medicine and Toxicology, Manipal Academy of Higher Education, Manipal, India

${ }^{445}$ Cochrane Unit, South African Medical Research Council, Cape Town, South Africa

${ }^{446}$ Department of General Surgery, Carol Davila University of Medicine and

Pharmacy, Bucharest, Romania

${ }^{447}$ Department of General Surgery, Emergency Hospital of Bucharest, Bucharest, Romania

${ }^{448}$ Department of Biological Sciences, University of Embu, Embu, Kenya

${ }^{449}$ Institute for Global Health Innovations, Duy Tan University, Hanoi, Vietnam

${ }^{450}$ Department of Pharmacology, Shahid Beheshti University of Medical Sciences, Tehran, Iran

${ }^{451}$ Heidelberg University Hospital, Heidelberg, Germany

${ }^{452}$ Public Health Department, Universitas Negeri Semarang, Kota Semarang, Indonesia

${ }^{453}$ Graduate Institute of Biomedical Informatics, Taipei Medical University, Taipei City, Taiwan

${ }^{454}$ School of Public Health and Family Medicine, University of Cape Town, Cape Town, South Africa

${ }^{455}$ Faculty of Medicine \& Health Sciences, Stellenbosch University, Cape Town, South Africa

${ }^{456}$ Reproductive Health Sciences, Department Obstetrics and Gynecology, University of Ibadan, Ibadan, Nigeria

${ }^{457}$ Department of Preventive Medicine, Kyung Hee University, Dongdaemun-gu, South Korea

${ }^{458}$ Disease Surveillance and Epidemic Response, Ministry of Health, Nairobi, Kenya

${ }^{459}$ Department of Psychiatry and Behavioural Neurosciences, McMaster University, Hamilton, ON, Canada

${ }^{460}$ Department of Psychiatry, University of Lagos, Lagos, Nigeria

${ }^{461}$ Department of Pathology and Molecular Medicine, McMaster University, Hamilton, ON, Canada

${ }^{462}$ Diplomacy and Public Relations Department, University of Human Development, Sulaimaniyah, Iraq

${ }^{463}$ Department of Pharmacology and Therapeutics, University of Nigeria Nsukka, Enugu, Nigeria

${ }^{464}$ Department of Psychology, University of Ghana, Accra, Ghana

${ }^{465}$ Discipline of Psychology, University of KwaZulu-Natal, Durban, South Africa

${ }^{466}$ Applied Research Division, Public Health Agency of Canada, Ottawa, ON, Canada

${ }^{467}$ School of Psychology, University of Ottawa, Ottawa, ON, Canada

${ }^{468}$ Department of Global Health Nursing, St Luke's International University, Chuo-ku,

${ }_{469}^{\text {Japan }}$ Academic department, Unium Ltd, Moscow, Russia

${ }^{470}$ Department of Project Management, National Research University Higher School

of Economics, Moscow, Russia

${ }^{471}$ Department of Respiratory Medicine, Jagadguru Sri Shivarathreeswara Academy of Health Education and Research, Mysore, India

${ }^{472}$ Department of Forensic Medicine, Manipal Academy of Higher Education, Manipal, India

${ }^{473}$ Department of Medicine, Ottawa Hospital Research Institute, OttawaON, Canada

${ }^{474}$ Parasitology and Mycology, Shiraz University of Medical Sciences, Shiraz, Iran

${ }^{475}$ Augenpraxis Jonas, Heidelberg University, Heidelberg, Germany

${ }^{476}$ Department of Medical Humanities and Social Medicine, Kosin University, Busan,

South Korea

${ }^{477}$ Research and Evaluation, Population Council, New Delhi, India

${ }^{478}$ Indian Institute of Health Management Research University Delhi, Jaipur, India

${ }^{479}$ Department of Pediatircs, RD Gardi Medical College, Ujjain, India

${ }^{480}$ Regional Medical Research Centre, Indian Council of Medical Research, Bhubaneswar, India

${ }^{481}$ Department of Paediatrics, University of Melbourne, Melbourne, VIC, Australia

${ }^{482}$ Population Health Theme, Murdoch Childrens Research Institute, Melbourne, VIC, Australia

${ }^{483}$ Department of Midwifery, Wolaita Sodo University, Wolaita Sodo, Ethiopia

${ }^{484}$ School of Public Health and Community Medicine, Faculty of Medicine, University

of New South Wales, Sydney, NSW, Australia

${ }^{485}$ Center for Research and Innovation, Ateneo De Manila University, Pasig City,

Philippines

${ }^{486}$ Department of Orthopedics, Yenepoya Medical College, Mangalore, India

${ }^{487}$ Shanghai Mental Health Center, Shanghai Jiao Tong University, Shanghai, China

${ }^{488}$ Department of Psychiatry, Department of Epidemiology, Columbia University, New York City, NY, USA

${ }^{489}$ Department of Chemistry, Faculty of Pharmacy, University of Porto, Porto, Portugal
${ }^{490}$ Department of Epidemiology and Evidence-Based Medicine, Sechenon University, Moscow, Russia

${ }^{491}$ Department of Nephrology, Sanjay Gandhi Postgraduate Institute of Medical Sciences, Lucknow, India

${ }^{492}$ Health Sciences Department, Muhammadiyah University of Surakarta, Sukoharjo, Indonesia

${ }^{493}$ Department of Population Studies, International Institute for Population Sciences, Mumbai, India

${ }^{494}$ Biomedical Engineering Department, Amirkabir University of Technology, Tehran, Iran

${ }^{495}$ Department of Chemistry, Sharif University of Technology, Tehran, Iran

${ }^{496}$ College of Medicine, University of Central Florida, Orlando, FL, USA

${ }^{497}$ College of Graduate Health Sciences, A T Still University, Mesa, AZ, USA

${ }^{498}$ Department of Epidemiology \& Biostatistics, Contech School of Public Health, Lahore, Pakistan

${ }^{499}$ Department of Medicine, University of Alberta, EdmontonAB, Canada

${ }^{500}$ Department of Immunology, Mazandaran University of Medical Sciences, Sari, Iran

${ }^{501}$ Molecular and Cell Biology Research Center, Mazandaran University of Medical

Sciences, Sari, Iran

${ }^{502}$ Thalassemia and Hemoglobinopathy Research Center, Ahvaz Jundishapur

University of Medical Sciences, Ahvaz, Iran

${ }^{503}$ Metabolomics and Genomics Research Center, Tehran University of Medical

Sciences, Tehran, Iran

${ }^{504}$ Faculty of Medicine, Mazandaran University of Medical Sciences, Sari, Iran

${ }^{505}$ Sina Trauma and Surgery Research Center, Tehran University of Medical Sciences, Tehran, Iran

${ }^{506}$ School of Nursing and Healthcare Professions, Federation University Australia,

Berwick, VIC, Australia

${ }^{507}$ School of Nursing and Midwifery, La Trobe University, Melbourne, VIC, Australia

${ }^{508}$ Faculty of Medicine, Birjand University of Medical Sciences, Birjand, Iran

${ }^{509}$ European Office for the Prevention and Control of Noncommunicable Diseases,

World Health Organization (WHO), Moscow, Russia

${ }^{510}$ Department of Surgery, University of Michigan, Ann Arbor, MI, USA

${ }^{511}$ Department of Oral Pathology, Srinivas Institute of Dental Sciences, Mangalore, India

${ }^{512}$ School of Behavioral Sciences and Mental Health, Tehran Institute of Psychiatry,

Tehran, Iran

${ }^{513}$ Forensic Medicine, Manipal Academy of Higher Education, Mangalore, India

${ }^{514}$ Kasturba Medical College, Manipal Academy of Higher Education, Mangalore, India

${ }_{515}^{5}$ Academic Public Health Department, Public Health England, London, UK

${ }^{516}$ School of Health, Medical and Applied Sciences, CQ University, Sydney, NSW, Australia

${ }^{517}$ Department of Computer Science, Metropolitan College, Boston University, Boston, USA

${ }^{518}$ Neurology Department, Sree Chitra Tirunal Institute for Medical Sciences and

Technology, Thiruvananthapuram, India

${ }^{519}$ School of Social Sciences and Psychology, Western Sydney University, Penrith, NSW, Australia

${ }^{520}$ Translational Health Research Institute, Western Sydney University, Penrith, NSW, Australia

${ }^{521}$ Brien Holden Vision Institute, Sydney, NSW, Australia

${ }^{522}$ Organization for the Prevention of Blindness, Paris, France

${ }^{523}$ EPIUnit - Public Health Institute University Porto (ISPUP), University of Porto, Porto, Portugal

${ }^{524}$ Surgery Department, University of Minnesota, Minneapolis, MN, USA

${ }^{525}$ Surgery Department, University Teaching Hospital of Kigali, Kigali, Rwanda

${ }^{526}$ Research Directorate, Nihon Gakko University, Fernando de la Mora, Paraguay

${ }^{527}$ Research Direction, Universidad Nacional de Caaguazú, Coronel Oviedo, Paraguay

${ }^{528}$ Golestan Research Center of Gastroenterology and Hepatology, Golestan

University of Medical Sciences, Gorgan, Iran

${ }^{529}$ Faculty of Medicine, Ain Shams University, Cairo, Egypt

${ }^{530}$ National Institute for Research in Environmental Health, Indian Council of Medical

Research, Bhopal, India

${ }^{53}$ Department of Epidemiology, Shahid Beheshti University of Medical Sciences,

Tehran, Iran

${ }^{532}$ College of Medicine, University of Sharjah, Sharjah, United Arab Emirates

${ }^{533}$ Emergency Department, Shahid Beheshti University of Medical Sciences, Tehran, Iran

${ }^{534}$ Faculty of Public Health, Kermanshah University of Medical Sciences, Kermanshah, Iran

${ }^{535}$ Department of Health in Disasters and Emergencies, Shahid Beheshti University of

Medical Sciences, Tehran, Iran

${ }^{536}$ Department of Neuroscience, Iran University of Medical Sciences, Tehran, Iran

${ }^{537}$ Nanobiotechnology Center, Soran University, Soran, Iraq

${ }^{538}$ Taleghani Hospital, Kermanshah University of Medical Sciences, Kermanshah, Iran

${ }^{539}$ Radiology and Nuclear Medicine Department, Kermanshah University of Medical Sciences, Kermanshah, Iran 
${ }^{540}$ Research Deputy, Taleghani Hospital, Kermanshah, Iran

${ }^{541}$ Public Health and Community Medicine Department, Cairo University, Giza, Egypt

${ }^{542}$ Department of Urology, Cairo University, Cairo, Egypt

${ }^{543}$ Public Health and Policy, London School of Hygiene \& Tropical Medicine, London, UK

${ }^{544} \mathrm{Global}$ Health Institute, American University of Beirut, Beirut, Lebanon

${ }^{545}$ Health and Disability Intelligence Group, Ministry of Health, Wellington, New Zealand

${ }^{546}$ Department of Entomology, Ain Shams University, Cairo, Egypt

${ }^{547}$ Department of Surgery, Marshall University, Huntington, WV, USA

${ }^{548}$ Department of Nutrition and Preventive Medicine, Case Western Reserve University, Cleveland, $\mathrm{OH}$, USA

${ }^{549}$ Rheumatology Department, University Hospitals Bristol NHS Foundation Trust, Bristol, UK

${ }^{550}$ Institute of Bone and Joint Research, University of Sydney, Syndey, NSW, Australia

${ }^{551}$ Institute of Social Medicine, University of Belgrade, Belgrade, Serbia

${ }^{552}$ Centre-School of Public Health and Health Management, University of Belgrade, Belgrade, Serbia

${ }^{553}$ Health Economics, Bangladesh Institute of Development Studies (BIDS), Dhaka, Bangladesh

${ }^{554}$ Colorectal Research Center, Iran University of Medical Sciences, Tehran, Iran

${ }^{555}$ Surgery Department, Hamad Medical Corporation, Doha, Qatar

${ }^{556}$ Faculty of Health \& Social Sciences, Bournemouth University, Bournemouth, UK

${ }^{557}$ Department of Public Health Sciences, University of North Carolina at Charlotte, Charlotte, NC, USA

${ }^{558}$ Department of Paediatrics, University of Melbourne, Parkville, VIC, Australia

${ }^{559}$ Centre for Adolescent Health, Murdoch Childrens Research Institute, Parkville, VIC Australia

${ }^{560}$ School of Public Health, Imperial College London, London, UK

${ }^{561}$ Faculty member of Education Development Center, Ahvaz Jundishapur University

of Medical Sciences, Ahvaz, Iran

${ }^{562}$ Department of Psychology, University of Alabama at Birmingham, Birmingham,

$A L$, USA

${ }^{563}$ Department of Psychiatry, Stellenbosch University, Cape Town, South Africa

${ }^{564}$ Emergency Department, Manian Medical Centre, Erode, India

${ }^{565}$ Microbiology Service, National Institutes of Health, Bethesda, MD, USA

${ }^{566}$ Center for Biomedical Information Technology, Shenzhen Institutes of Advanced

Technology, Chinese Academy of Sciences, Shenzhen, China

${ }^{567}$ Department of Health Promotion and Education, Alborz University of Medical

Sciences, Karaj, Iran

${ }^{568}$ Health Policy Research Center, Shiraz University of Medical Sciences, Shiraz, Iran

${ }^{569}$ Independent Consultant, Karachi, Pakistan

${ }^{570}$ School of Medicine, Alborz University of Medical Sciences, Karaj, Iran

${ }^{571}$ Chronic Diseases (Home Care) Research Center, Hamadan University of Medical Sciences, Hamadan, Iran

${ }^{572}$ HIV/STI Surveillance Research Center, and WHO Collaborating Center for HIV

Surveillance, Institute for Futures Studies in Health, Kerman University of Medical

Sciences, Kerman, Iran

${ }^{573}$ Centre for Medical Informatics, University of Edinburgh, Edinburgh, UK

${ }^{574}$ Division of General Internal Medicine, Harvard University, Boston, MA, USA

${ }^{575}$ National Institute of Infectious Diseases, Tokyo, Japan

${ }^{576}$ College of Medicine, Yonsei University, Seodaemun-gu, South Korea

${ }^{577}$ Division of Cardiology, Emory University, Atlanta, GA, USA

${ }^{578}$ Finnish Institute of Occupational Health, Helsinki, Finland

${ }^{579}$ Department of Health Education \& Promotion, Kermanshah University of Medical Sciences, Kermanshah, Iran

${ }^{580}$ School of Health, University of Technology Sydney, Sydney, NSW, Australia

${ }^{581}$ Department of Psychology, Reykjavik University, Reykjavik, Iceland

${ }^{582}$ Department of Health and Behavior Studies, Columbia University, New York, NY, USA

${ }^{583}$ Department of Forensic Medicine, Kathmandu University, Dhulikhel, Nepal

${ }^{584}$ Department of Medicine, University of Alabama at Birmingham, Birmingham, AL, USA

${ }^{585}$ Medicine Service, US Department of Veterans Affairs (VA), Birmingham, AL, USA

${ }^{586}$ Department of Epidemiology, School of Preventive Oncology, Patna, India

${ }^{587}$ Department of Epidemiology, Healis Sekhsaria Institute for Public Health, Mumbai, India

${ }^{588}$ 2nd Department of Surgery-SUUB, Carol Davila University of Medicine and

Pharmacy, Bucharest, Romania

${ }^{589}$ 2nd Surgery Department, Bucharest Emergency Hospital, Bucharest, Romania

${ }^{590}$ Pain Management Research Institute (PMRI), Northern Clinical School, University of Sydney, St Leonards, NSW, Australia

${ }^{591}$ Michael J Cousins Pain Management \& Research Centre, Northern Sydney Loca Health District, St Leonards, NSW, Australia

${ }^{592}$ Department of Medical Surgical Nursing, Urmia University of Medical Science, Urmia, Iran

${ }^{593}$ Emergency Nursing Department, Semnan University of Medical Sciences, Semnan, Iran
${ }^{594}$ Department of Biostatistics, Hamedan University of Medical Sciences, Hamadan, Iran

${ }^{595}$ Hospital Universitario de la Princesa, Autonomous University of Madrid, Madrid, Spain

${ }^{596}$ Centro de Investigación Biomédica en Red Enfermedades Respiratorias (CIBERES), Madrid, Spain

${ }^{597}$ Department of Public Health, Arba Minch University, Arba Minch, Ethiopia

${ }^{598}$ Hull York Medical School, University of Hull, Hull City, UK

${ }^{599}$ Usher Institute of Population Health Sciences and Informatics, University of Edinburgh, Edinburgh, UK

${ }^{600}$ Department of Psychiatry and Mental Health, University of Cape Town, Cape

Town, South Africa

${ }^{601}$ South African Medical Research Council, Cape Town, South Africa

${ }^{602}$ Department of Psychology, Deakin University, Burwood, VIC, Australia

${ }^{603}$ Department of Community Medicine, Ahmadu Bello University, Zaria, Nigeria

${ }^{604}$ Department of Agriculture and Food Systems, University of Melbourne, Melbourne,

VIC, Australia

${ }^{605}$ Department of Criminology, Law and Society, University of California Irvine, Irvine,

CA, USA

${ }^{606}$ Department of Medicine, University of Valencia, Valencia, Spain

${ }^{607}$ Carlos III Health Institute, Biomedical Research Networking Center for Mental

Health Network (CiberSAM), Madrid, Spain

${ }^{608}$ School of Social Work, University of Illinois, Urbana, IL, USA

${ }^{609}$ Department of Public Health, Arbaminch College of Health Sciences, Arbaminch town sikela, Ethiopia

${ }^{610}$ Axum College of Health Science, mekelle, Ethiopia

${ }^{611}$ School of Midwifery, University of Gondar, Gondar, Ethiopia

${ }^{612} \mathrm{School}$ of Public Health, University of Adelaide, Adelaide, SA, Australia

${ }^{613}$ Department of Environmental Health, Wollo University, Dessie, Ethiopia

${ }^{614}$ Department of Community and Family Medicine, Iran University of Medical

Sciences, Tehran, Iran

${ }^{615}$ Department of Pharmacognosy, Mekelle University, Mekelle, Ethiopia

${ }^{616}$ Institute of Public Health, University of Gondar, Gondar, Ethiopia

${ }^{617}$ Department of Public Health, Adigrat University, Adigrat, Ethiopia

${ }^{618}$ Psychiatry Department, Hawassa University, Hawassa, Ethiopia

${ }^{619}$ Institute of Public Health, Jagiellonian University Medical College, Krakow, Poland

${ }^{620}$ The Agency for Health Technology Assessment and Tariff System, Warszawa, Poland

${ }^{621}$ Department of Health Economics, Hanoi Medical University, Hanoi, Vietnam

${ }^{622}$ Department of Molecular Medicine and Pathology, University of Auckland,

Auckland, New Zealand

${ }^{623}$ Clinical Hematology and Toxicology, Military Medical University, Hanoi, Vietnam

${ }^{624}$ Department of Community Medicine, All India Institute of Medical Sciences,

Nagpur, India

${ }^{625}$ Department of Psychiatry, Massachusetts General Hospital, Boston, MA, USA

${ }^{626} \mathrm{Mbarara}$ University of Science and Technology, Mbarara, Uganda

${ }^{627}$ Lee Kong Chian School of Medicine, Nanyang Technological University, Singapore

${ }^{628}$ Institute of Soil and Environmental Sciences, A T Still University, Faisalabad,

Pakistan

${ }^{629} \mathrm{Gomal}$ Center of Biochemistry and Biotechnology, Gomal University, Dera Ismail

Khan, Pakistan

${ }^{630}$ TB Culture Laboratory, Mufti Mehmood Memorial Teaching Hospital, Dera Ismail

Khan, Pakistan

${ }^{631}$ Research Department, National Institute of Population Studies (NIPS), Islamabad, Pakistan

${ }^{632}$ Amity Institute of Biotechnology, Amity University Rajasthan, Jaipur, India

${ }^{633}$ Division of Health Sciences, University of Warwick, Coventry, UK

${ }^{634}$ Argentine Society of Medicine, Buenos Aires, Argentina

${ }^{635}$ Velez Sarsfield Hospital, Buenos Aires, Argentina

${ }^{636}$ UKK Institute, Tampere, Finland

${ }^{637}$ Raffles Neuroscience Centre, Raffles Hospital, Singapore

${ }^{638}$ Yong Loo Lin School of Medicine, National University of Singapore, Singapore

${ }^{639}$ Department of Medical and Surgical Sciences, University of Bologna, Bologna, Italy

${ }^{640}$ Occupational Health Unit, Sant'Orsola Malpighi Hospital, Bologna, Italy

${ }^{641}$ Department of Health Care Administration and Economics, National Research

University Higher School of Economics, Moscow, Russia

${ }^{642}$ Foundation University Medical College, Foundation University, Islamabad, Pakistan

${ }^{643}$ Demographic Change and Ageing Research Area, Federal Institute for Population

Research, Wiesbaden, Germany

${ }^{644}$ Center of Population and Health, Wiesbaden, Germany

${ }^{645}$ Department of Physical Therapy, Naresuan University, Meung District, Thailand

${ }^{646}$ Department of Human Anatomy, Histology, Embryology, Bahir Dar University,

Bahir Dar, Ethiopia

${ }^{647}$ Department of Pharmacology and Toxicology, Mekelle University, Mekelle, Ethiopia

${ }^{648}$ Department of Pharmacology, Addis Ababa University, Addis ababa, Ethiopia

${ }^{649}$ Department of Nursing, Wollo University, Dessie, Ethiopia

${ }^{650}$ Department of Orthopaedics, Wenzhou Medical University, Wenzhou, China 
${ }^{651}$ Medical Physics Department, Ahvaz Jundishapur University of Medical Sciences, Ahvaz, Iran

${ }^{652}$ Department of Preventive Medicine, Northwestern University, Chicago, IL, USA ${ }^{653}$ School of International Development and Global Studies, University of Ottawa, OttawaON, Canada

${ }^{654}$ Health Services Management Research Center, Kerman University of Medical Sciences, Kerman, Iran

${ }^{655}$ Department of Health Management, Policy and Economics, Kerman University of Medical Sciences, Kerman, Iran

${ }^{656} \mathrm{Centre}$ for Suicide Research and Prevention, University of Hong Kong, Hong Kong, China

${ }^{657}$ Department of Social Work and Social Administration, University of Hong Kong, Hong Kong, China

${ }^{658}$ School of Allied Health Sciences, Addis Ababa University, Addis Ababa, Ethiopia

${ }^{659}$ Department of Psychopharmacology, National Center of Neurology and Psychiatry, Tokyo, Japan

${ }^{660}$ Department of Sociology, Yonsei University, Seoul, South Korea

${ }^{661}$ Department of Health Policy \& Management, Jackson State University, Jackson, MS, USA

${ }^{662}$ School of Medicine, Tsinghua University, Beijing, China

${ }^{663}$ Department of Environmental Health, Mazandaran University of Medical Sciences, Sari, Iran

${ }^{664}$ Department of Environmental Health, Academy of Medical Science, Sari, Iran

${ }^{665}$ School of Public Health and Management, Hubei University of Medicine, Shiyan, China

${ }^{666}$ Department of Epidemiology and Biostatistics, Wuhan University, Wuhan, China

${ }^{667}$ Global Health Institute, Wuhan University, Wuhan, China

${ }^{668}$ Social Determinants of Health Research Center, Ardabil University of Medical

Science, Ardabil, Iran

${ }^{669}$ Department of Epidemiology, University Hospital of Setif, Setif, Algeria

${ }^{670}$ Department of Medicine, School of Clinical Sciences at Monash Health, Monash University, Melbourne, VIC, Australia

${ }^{671}$ Student Research Committee, Babol University of Medical Sciences, Babol, Iran

${ }^{672}$ Department of Community Medicine, Ardabil University of Medical Science, Ardabil, Iran

${ }^{673}$ Department of Environment Health Engineering, Gonabad University of Medical Sciences, Gonabad, Iran

${ }^{674}$ Faculty of Medical Sciences, Department of Health Education, Tarbiat Modares University, Tehran, Iran

${ }^{675}$ Department of Preventive Medicine, Wuhan University, Wuhan, China

${ }^{676}$ School of Public Health, Wuhan University of Science and Technology, Wuhan, China

${ }^{677}$ Hubei Province Key Laboratory of Occupational Hazard Identification and Control, Wuhan University of Science and Technology, Wuhan, China

${ }^{678}$ Indian Institute of Public Health, Public Health Foundation of India, Gurugram, India

${ }^{679}$ National Drug and Alcohol Research Centre, University of New South Wales, Sydney, NSW, Australia

${ }^{680}$ Department of Community Medicine, University of Peradeniya, Peradeniya, $\mathrm{Sr}$ Lanka

${ }^{681}$ Faculty of Infectious and Tropical Diseases, London School of Hygiene \& Tropical Medicine, London, UK

Acknowledgements Syed Aljunid acknowledges the Department of Health Policy and Management, Faculty of Public Health, Kuwait University and International Centre for Casemix and Clinical Coding, Faculty of Medicine, National University of Malaysia and for the approval and support to participate in this research project. Alaa Badawi acknowledges support from the Public Health Agency of Canada. Till Bärnighausen acknowledges support from the Alexander von Humboldt Foundation through the Alexander von Humboldt Professor award, funded by the German Federal Ministry of Education and Research. Felix Carvalho acknowledges UID/ MULTI/04378/2019 support with funding from FCT/MCTES through national funds. Vera M Costa acknowledges her grant (SFRH/BHD/110001/2015), received by Portuguese national funds through Fundação para a Ciência e Tecnologia (FCT), IP, under the Norma Transitória DL57/2016/CP1334/CT0006. Kebede Deribe acknowledges support from a grant from the Wellcome Trust [grant number 201900] as part of his International Intermediate Fellowship. Tim Driscoll acknowledges the work on occupational risk factors was partially supported by funds from the World Health Organization. Eduarda Fernandes acknowledges UID/ QUI/50006/2019 support with funding from FCT/MCTES through national funds. Yuming Guo acknowledges support from Career Development Fellowships of the Australian National Health and Medical Research Council (numbers APP1107107 and APP1163693). Sheikh Mohammed Shariful Islam acknowledges funding by a Fellowship from National Heart Foundation of Australia and Institute for Physical Activity and Nutrition, Deakin University.Mihajlo Jakovljevic acknowledges support by the Ministry of Education Science and Technological Development of the Republic of Serbia through the Grant number Ol175014; publication of results was not contingent upon Ministry's censorship or approval. Sudha Jayaraman acknowledges support from: NIH R21: 1R21TW010439-01A1 (PI); Rotary Foundation Global Grant \#GG1749568 (PI); NIH P20: 1P20CA210284-01A1 (Co-PI); DOD grant W81XWH-16-2-0040 (Co-I) during the submitted work. Yun Jin Kim acknowledges support from a grant from the Research Management Centre, Xiamen University Malaysia [grant number: XMUMRF/2018-C2/ITCM/0001]. Kewal Krishan acknowledges support by UGC Centre of Advanced Study (CAS II) awarded to the Department of Anthropology, Panjab University, Chandigarh, India. Manasi Kumar acknowledges FIC/NIH funding from grant K43 1K43MH114320-01. Amanda Mason-Jones acknowledges institutional support from the University of York. Walter Mendoza is currently Program Analyst Population and Development at the Peru Country Office of the United Nations Population Fund-UNFPA, which not necessarily endorses this study. Mariam Molokhia acknowledges support from the National Institute for Health Research Biomedical Research Center at Guy's and St Thomas' National Health Service Foundation Trust and King's College London. Ilais Moreno Velásquez acknowledges support by the Sistema Nacional de Investigación (SNI, Senacyt, Panama). Mukhammad David Naimzada acknowledges support from Government of the Russian Federation (Agreement No - 075-022019-967). Stanislav S. Otstavnov acknowledges the support from the Government of the Russian Federation (Agreement No - 075-02-2019-967). Ashish Pathak acknowledges support from the Indian Council of Medical Research (ICMR), New Delhi, India (Grant number 2013-1253). Michael R Phillips acknowledges support in part by a grant from the National Science Foundation of China (No. 81761128031). Marina Pinheiro acknowledges FCT for funding support through program DL 57/2016-Norma transitória. Abdallah M. Samy acknowledges support from a fellowship from the Egyptian Fulbright Mission Program. Milena Santric Milicevic acknowledges the support from the Ministry of Education, Science and Technological Development, the Republic of Serbia (Contract No. 175087). Seyedmojtaba Seyedmousavi acknowledges support from the Intramural Research Program of the National Institutes of Health, Clinical Center, Department of Laboratory Medicine, Bethesda, MD, USA. Rafael Tabarés-Seisdedos acknowledges support in part by the national grant PI17/00719 from ISCIII-FEDER. Sojib Bin Zaman acknowledges support from an "Australian Government Research Training Program (RTP) Scholarship." Louisa Degenhardt acknowledges support from an Australian National Health and Medical Research Council (NHMRC) Senior Principal Research Fellowship (\#1135991) and by a National Institute of Health (NIH) National Institute on Drug Abuse (NIDA) grant (R01DA1104470).

Funding Bill and Melinda Gates Foundation OPP1152504.

Map disclaimer The depiction of boundaries on the map(s) in this article do not imply the expression of any opinion whatsoever on the part of BMJ (or any member of its group) concerning the legal status of any country, territory, jurisdiction or area or of its authorities. The map(s) are provided without any warranty of any kind, either express or implied.

Competing interests Dr. James reports grants from Sanofi Pasteur, outside the submitted work. Dr. Driscoll reports grants from World Health Organization, during the conduct of the study. Dr. Ivers reports grants from National Health and Medical Research Council of Australia, during the conduct of the study. Dr. Jozwiak reports personal fees from TEVA, personal fees from ALAB, personal fees from BOEHRINGER INGELHEIM, personal fees from SYNEXUS, non-financial support from SERVIER, nonfinancial support from MICROLIFE, non-financial support from MEDICOVER, outside the submitted work. Dr. Rakovac reports grants from World Health Organization, during the conduct of the study. Dr Shariful Islam is funded by National Heart Foundation of Australia and Institute for Physical Activity and Nutrition, Deakin University. Dr. Sheikh reports grants from Health Data Research UK, outside the submitted work. Dr. Singh reports personal fees from Crealta/Horizon, Medisys, Fidia, UBM LLC, Trio health, Medscape, WebMD, Clinical Care options, Clearview healthcare partners, Putnam associates, Spherix, Practice Point communications, the National Institutes of Health and the American College of Rheumatology, and Speaker's bureau of Simply Speaking, owns stock options in Amarin pharmaceuticals and Viking pharmaceuticals, serves on the steering committee of OMERACT, an international organization that develops measures for clinical trials and receives arm's length funding from 12 pharmaceutical companies, serves on the FDA Arthritis Advisory Committee, is a member of the Veterans Affairs Rheumatology Field Advisory Committee, and is the editor and the Director of the UAB Cochrane Musculoskeletal Group Satellite Center on Network Meta-analysis, outside the submitted work. Dr. Stein reports personal fees from Lundbeck, personal fees from Sun, outside the submitted work. Dr. Degenhardt reports grants from Indivior, Seqirus, Reckitt Benckiser, outside the submitted work.

Patient consent for publication Not required. 
Provenance and peer review Not commissioned; externally peer reviewed.

Data availability statement Availability of input data depends on original source. Select data are available in a public, open access repository. Select data are available on reasonable request. Select data may be obtained from a third party and are not publicly available. All results relevant to the study are included in the article or uploaded as supplementary information or are available online.

Open access This is an open access article distributed in accordance with the Creative Commons Attribution 4.0 Unported (CC BY 4.0) license, which permits others to copy, redistribute, remix, transform and build upon this work for any purpose, provided the original work is properly cited, a link to the licence is given, and indication of whether changes were made. See: https://creativecommons.org/ licenses/by/4.0/.

\section{REFERENCES}

1 GBD 2016 Traumatic Brain Injury and Spinal Cord Injury Collaborators. Global, regional, and national burden of traumatic brain injury and spinal cord injury, 19902016: a systematic analysis for the global burden of disease study 2016. Lancet Neurol 2016;18:56-87.

2 Kotagal M, Agarwal-Harding KJ, Mock C, et al. Health and economic benefits of improved injury prevention and trauma care worldwide. PLoS One 2014;9:e91862.

3 Sunshine JE, Meo N, Kassebaum NJ, et al. Association of adverse effects of medical treatment with mortality in the United States: a secondary analysis of the global burden of diseases, injuries, and risk factors study. JAMA Netw Open 2019;2:e187041.

4 Dandona R, Kumar GA, Kharyal A, et al. Mortality due to snakebite and other venomous animals in the Indian state of Bihar: findings from a representative mortality study. PLoS One 2018;13:e0198900.

5 Jha AK, Larizgoitia I, Audera-Lopez C, et al. The global burden of unsafe medical care: Analytic modelling of observational studies. BMJ Qual Saf 2013;22:809-15.

6 Goal 3. sustainable development knowledge platform. Available: https://sustainabled evelopment.un.org/sdg3 [Accessed 29 Sep 2019].

7 Wong E, Leong MKF, Anantharaman V, et al. Road traffic accident mortality in Singapore. J Emerg Med 2002;22:139-46.

8 Hsiao M, Malhotra A, Thakur JS, et al. Road traffic injury mortality and its mechanisms in India: nationally representative mortality survey of 1.1 million homes. BMJ Open 2013;3:e002621.

9 Montazeri A. Road-traffic-related mortality in Iran: a descriptive study. Public Health 2004;118:110-3.

10 Ohlin $\mathrm{M}$, Algurén $\mathrm{B}$, Lie A. Analysis of bicycle crashes in Sweden involving injuries with high risk of health loss. Traffic Inj Prev 2019;20:613-8.

11 Haagsma JA, Graetz N, Bolliger I, et al. The global burden of injury: incidence, mortality, disability-adjusted life years and time trends from the global burden of disease study 2013. Injury Prevention 2015:injuryprev-2015-041616.

12 Dicker D, Nguyen G, Abate D, et al. Global, regional, and national age-sex-specific mortality and life expectancy, 1950-2017: a systematic analysis for the global burden of disease study 2017. The Lancet 2018;392:1684-735.

13 Roth GA, Abate D, Abate KH, et al. Global, regional, and national age-sex-specific mortality for 282 causes of death in 195 countries and territories, 1980-2017: a systematic analysis for the global burden of disease study 2017. The Lancet 2018;392:1736-88.

14 Stanaway JD, Afshin A, Gakidou E, et al. Global, regional, and national comparative risk assessment of 84 behavioural, environmental and occupational, and metabolic risks or clusters of risks for 195 countries and territories, 1990-2017: a systematic analysis for the global burden of disease study 2017. The Lancet 2018;392:1923-94.

$15 \mathrm{Kyu} \mathrm{HH}$, Abate D, Abate KH, et al. Global, regional, and national disability-adjusted life-years (DALYS) for 359 diseases and injuries and healthy life expectancy (HALE) for 195 countries and territories, 1990-2017: a systematic analysis for the global burden of disease study 2017. The Lancet 2018:392:1859-922.

16 James SL, Abate D, Abate KH, et al. Global, regional, and national incidence, prevalence, and years lived with disability for 354 diseases and injuries for 195 countries and territories, 1990-2017: a systematic analysis for the global burden of disease study 2017. The Lancet 2018;392:1789-858.

17 Murray CJL, Callender CSKH, Kulikoff XR, et al. Population and fertility by age and sex for 195 countries and territories, 1950-2017: a systematic analysis for the global burden of disease study 2017. The Lancet 2018;392:1995-2051.

18 Foreman KJ, Lozano R, Lopez AD, et al. Modeling causes of death: an integrated approach using CODEm. Popul Health Metr 2012;10:1.

19 Naghavi M, Makela S, Foreman K, et al. Algorithms for enhancing public health utility of national causes-of-death data. Popul Health Metr 2010;8:9.

20 Foreman KJ, Naghavi M, Ezzati M. Improving the usefulness of US mortality data: new methods for reclassification of underlying cause of death. Popul Health Metr 2016;14.

21 China Zhuhai Study 2006-2007 - China CDC | GHDx. Available: http://ghdx. healthdata.org/record/china-zhuhai-study-2006-2007-china-cdc [Accessed 15 May 2018].

22 Functional outcome at 2.5, 5, 9, and 24 months after injury in the Netherlands | GHDx. Available: http://ghdx. healthdata.org/record/functional-outcome-25-5-9-and24-months-after-injury-netherlands [Accessed 15 May 2018].

23 Health-Related quality of life after burns: a prospective multicentre cohort study with 18 months follow-up | GHDx. Available: http://ghdx.healthdata.org/record/healthrelated-quality-life-after-burns-prospective-multicentre-cohort-study-18-monthsfollow [Accessed 15 May 2018].

24 Netherlands injury surveillance system 2007 | GHDx. Available: http://ghdx. healthdata.org/record/netherlands-injury-surveillance-system-2007 [Accessed 15 May 2018].

25 Netherlands injury surveillance system 2010 | GHDx. Available: http://ghdx. healthdata.org/record/netherlands-injury-surveillance-system-2010 [Accessed 14 May 2018].

26 Mackenzie EJ, Rivara FP, Jurkovich GJ, et al. The national study on costs and outcomes of trauma. J Trauma 2007:63:S54-67.

27 Traumatic Brain Injury(TBI) Follow-Up Registry and Surveillance of TBI in the Emergency Department (ED); Notice of Availability of Funds. Federal Register, 2002. Available: https://www.federalregister.gov/documents/2002/05/08/02-11359/ traumatic-brain-injurytbi-follow-up-registry-and-surveillance-of-tbi-in-the-emergencydepartment-ed [Accessed 14 May 2018].

28 Salomon JA, Haagsma JA, Davis A, et al. Disability weights for the global burden of disease 2013 study. Lancet Glob Health 2015;3:e712-23.

29 Stevens GA, Alkema L, Black RE, et al. Guidelines for accurate and transparent health estimates reporting: the gather statement. The Lancet 2016;388:e19-23.

30 Lim SS, Updike RL, Kaldjian AS, et al. Measuring human capital: a systematic analysis of 195 countries and territories, 1990-2016. The Lancet 2018;392:1217-34.

31 Fann JR, Ribe AR, Pedersen HS, et al. Long-Term risk of dementia among people with traumatic brain injury in Denmark: a population-based observational cohort study. The Lancet Psychiatry 2018:5:424-31.

32 Who | global status report on road safety, 2018. Available: https://www.who.int/ violence_injury_prevention/road_safety_status/2018/en/ [Accessed 22 Jul 2019].

33 Safety 2020. Safety 2020. Available: https://www.worldsafety2020.com [Accessed 22 Jul 2019]. 
Correction: Global injury morbidity and mortality from 1990 to 2017: results from the Global Burden of Disease Study 2017

James SL, Castle CD, Dingels ZV, et al. Global injury morbidity and mortality from 1990 to 2017: results from the Global Burden of Disease Study 2017. Inj Prev. 2020. doi: 10.1136/ injuryprev-2019-043494

This article was previously published with errors in affiliations for authors Serge Resnikof and Aziz Rezapour. The updated affiliations are below

Serge Resnikoff 521,522

${ }^{521}$ Brien Holden Vision Institute, Sydney, NSW, Australia

${ }^{522}$ Organization for the Prevention of Blindness, Paris, France

Aziz Rezapour ${ }^{30}$

${ }^{30}$ Health Management and Economics Research Center, Iran University of Medical Sciences, Tehran, Iran

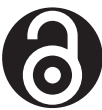

\section{OPEN ACCESS}

Open access This is an open access article distributed in accordance with the Creative Commons Attribution 4.0 Unported (CC BY 4.0) license, which permits others to copy, redistribute, remix, transform and build upon this work for any purpose, provided the original work is properly cited, a link to the licence is given, and indication of whether changes were made. See: https://creativecommons.org/licenses/by/4.0/.

(C) Author(s) (or their employer(s)) 2020. Re-use permitted under CC BY. Published by BMJ.

Inj Prev 2020;26:1165. doi:10.1136/injuryprev-2019-043494corr1

D) Check for updates 